\title{
The small and large intestine contain transcriptionally related mesenchymal stromal cell subsets that derive from embryonic Gli1+ mesothelial cells
}

\section{Simone Pærregaard}

Technical University of Denmark https://orcid.org/0000-0001-6879-0529

\section{Sophie Schussek}

Technical University of Denmark

\section{Line Wulff}

Technical University of Denmark https://orcid.org/0000-0002-7482-4569

\section{Kristoffer Niss}

University of Copenhagen

\section{Urs Mörbe}

Technical University of Denmark https://orcid.org/0000-0001-8747-437X

Johan Jendholm

Technical University of Denmark

\section{Kerstin Wendland}

Lund University

\section{Anna Andrusaite}

University of Glasgow

\section{Kevin Brulois}

Stanford University

\section{Robert Nibbs}

University of Glasgow https://orcid.org/0000-0002-8150-0044

\section{Katarzyna Sitnik}

Helmholtz Centre for Infection Research

\section{Allan Mowat}

University of Glasgow https://orcid.org/0000-0001-9389-3079

\section{Eugene Butcher}

Stanford University School of Medicine https://orcid.org/0000-0001-8786-7907

\section{Søren Brunak}

University of Copenhagen https://orcid.org/0000-0003-0316-5866

William Agace ( $\nabla$ william.agace@med.lu.se)

Technical University of Denmark 


\section{Article}

Keywords: Intestinal fibroblasts (FB), mesenchymal stromal cells (MSC), intestinal homeostasis Posted Date: October 8th, 2021

DOI: https://doi.org/10.21203/rs.3.rs-829151/v1

License: (c) (1) This work is licensed under a Creative Commons Attribution 4.0 International License. Read Full License 
2 The small and large intestine contain transcriptionally related mesenchymal stromal cell subsets that derive from embryonic $\mathrm{Gli1}^{+}$mesothelial cells

5 Simone Isling Pærregaard ${ }^{1}$, Sophie Schussek ${ }^{1, \#}$, Line Wulff $^{1, \#}$, Kristoffer Niss $^{2}$, Urs Mörbe ${ }^{1}$,

6 Johan Jendholm ${ }^{1}$, Kerstin Wendland ${ }^{3}$, Anna T. Andrusaite ${ }^{4}$, Kevin F. Brulois ${ }^{5}$, Robert J. B.

7 Nibbs $^{4}$, Katarzyna Sitnik ${ }^{1}$, Allan McI Mowat ${ }^{4}$, Eugene C. Butcher ${ }^{5,6}$, Søren Brunak ${ }^{2}$, William 8 W. $\operatorname{Agace}^{1,3, *}$.

$10{ }^{1}$ Department of Health Technology, Technical University of Denmark, Kemitorvet, 2800

11 Kgs. Lyngby, Denmark.

$12 \quad{ }^{2}$ Novo Nordisk Foundation Center for Protein Research, Faculty of Health and Medical

13 Sciences, University of Copenhagen, Copenhagen 2200, Denmark.

$14{ }^{3}$ Immunology Section, Lund University, Lund 221 84, Sweden.

$15{ }^{4}$ Institute of Infection, immunity and Inflammation, University of Glasgow, Glasgow, 16 Scotland.

$17 \quad{ }^{5}$ Laboratory of Immunology and Vascular Biology, Department of Pathology, School of 18 Medicine, Stanford University, Stanford, USA.

$19{ }^{6}$ The Center for Molecular Biology and Medicine, Veterans Affairs Palo Alto Health Care 20 System and the Palo Alto Veterans Institute for Research (PAVIR), Palo Alto, USA

21

$22{ }^{\#}$ Equal contribution

23

$24 *$ Correspondence: William Agace, wiag@ dtu.dk 


\section{Abstract}

27 Intestinal fibroblasts (FB) play essential roles in intestinal homeostasis. Here we show that the small and large intestinal lamina propria (LP) contain similar FB subsets that locate in specific anatomical niches and express distinct arrays of epithelial support genes. However, there were tissue specific differences in the transcriptional profile of intestinal FB subsets in the two sites. All adult intestinal LP mesenchymal stromal cells (MSC), including FB, smooth muscle cells (SMC) and pericytes derive from Gli1-expressing embryonic precursors which we identify as mesothelial cells. Trajectory analysis suggested that adult SMC and FB derive from distinct embryonic intermediates, and that adult FB subsets develop in a linear trajectory from $\mathrm{CD} 81^{+}$FB. Finally, we show that colonic subepithelial PDGFR $\alpha^{\text {hi }}$ FB comprise several functionally and anatomically distinct populations that originate from an Fgfr2-expressing FB intermediate. Collectively our results provide novel insights into MSC diversity, location, function and ontogeny, with implications for our understanding of intestinal development, homeostasis and disease.

\section{Introduction}

42 The small and large intestines form a continuous tube from the stomach to the anus, but are functionally and anatomically distinct. The small intestine is the primary site of food digestion and nutrient absorption and is characterized by finger-like projections termed villi that protrude into the intestinal lumen and maximize the absorptive area of the epithelium. In contrast, the large intestine is primarily a site of water absorption and is a major niche for beneficial microbes; its surface consists of crypts linked by short regions of flat surface epithelium. The cellular composition of the intestinal mucosa also differs markedly between the small and large intestines ${ }^{1,2}$. For example, the small and large intestines contain different numbers and proportions of innate and adaptive immune cells as well as epithelial 
51

subpopulations ${ }^{1-4}$. These distinct segments are also exposed to different concentrations of microbial and food-derived metabolites that regulate the composition and function of local cells ${ }^{1,2}$. However, the cellular and signaling components that determine the differences in tissue structure and composition are not fully understood. mesenchymal stromal cells (MSC) that include fibroblasts (FB), pericytes (PC) and smooth muscle cells (SMCs) that play an essential role in intestinal homeostasis ${ }^{5-11}$. For example, intestinal FB are major producers of extracellular matrix proteins that help provide structure to the mucosa ${ }^{12,13}$. They also express factors essential for epithelial ${ }^{6-8,10,14,15}$ and endothelial homeostasis ${ }^{11,16,17}$, as well as immune cell localization and function ${ }^{18-21}$. Recent single cell (sc)RNA-seq studies have demonstrated considerable heterogeneity within the intestinal LP MSC compartment and have led to the identification of several FB clusters with nonredundant functions in intestinal homeostasis $5,7-9,11,22,23$. A picture is also emerging whereby different intestinal FB subsets locate within distinct regions of the mucosa $3,6,7,10,11,16,17$, providing specialized support to cells in their local environment ${ }^{5-8,10,11,14,15,24}$. However, the exact nature of these diverse LP MSC subsets and how they differ in the small and large intestine remains to be established. scRNA-seq analyses have shown that the composition of human intestinal MSC populations changes markedly as the tissue develops in the embryo ${ }^{17,25}$. Although the origin of these populations remains to be determined, lineage-tracing experiments in mice have suggested that the mesothelium, an epithelial monolayer that lines the serosal surface of the intestine $^{26}$, can give rise to SMC and various FB in the intestinal serosa and muscle layers ${ }^{27,28}$. Whether MSC subsets present within the adult intestinal LP derive from cells of 
76 common or distinct embryonic origin and the developmental relationship between adult MSC

77 subsets remains unclear.

78 Here we demonstrate that LP MSC subset composition is similar in the small and 79 large intestine and that each subset occupies distinct anatomical niches. Nevertheless, the 80 transcriptional profile of the major LP FB subsets differed markedly between the small and 81 large intestine, suggesting regional specific functions in intestinal homeostasis. Grafting and 82 lineage-tracing experiments demonstrated that all MSC subsets in adult small intestinal and 83 colonic LP derive from Glil-expressing precursors present in embryonic day (E)12.5

84 intestine. Computational analysis suggested that all adult intestinal MSC derive from 85 embryonic intestinal mesothelial cells and that adult SMC and FB arise from distinct 86 mesothelial derived embryonic intermediates. We could also define a linear developmental 87 trajectory for all adult FB subsets that originated from $\mathrm{CD} 81^{+} \mathrm{FB}$. 
Results

The small intestine and colon LP contain diverse, but transcriptionally related MSC subsets.

To gain a broad understanding of MSC subset diversity in the intestinal LP, we performed scRNA-seq on MSC isolated from the small intestine and colon LP of 8-10 week old mice. Briefly, after removal of Peyer's patches, muscularis externa and epithelium, intestinal MSCs were enriched from digested intestinal LP cell suspensions by fluorescently activated cell sorting of live, single, lineage $\left(\mathrm{CD} 45^{+}\right.$, Ter119 $\left.{ }^{+}\right)$, non-epithelial $\left(\mathrm{EpCAM}^{+}\right)$, non-endothelial $\left(\mathrm{CD} 31^{+}\right)$, non-lymphoid tissue-associated MSCs $\left(\mathrm{BP}^{+}\right)^{29}$ and non-glial cells $\left(\mathrm{L}^{2} \mathrm{CAM}{ }^{+}\right)$, followed by gating on cells expressing the pan MSC marker Itg $\beta 1$ (Supplementary Fig. 1A). After bioinformatic removal of contaminating c-kit ${ }^{+}$interstitial cells of Cajal (ICC), CD31 ${ }^{+}$ endothelial cells, plasma cells and CD45 ${ }^{+}$immune cells, sequencing data of 16.964 small intestinal and 14.164 colonic MSC remained.

Louvain clustering identified six small intestinal MSC clusters (Fig. 1A) and differential gene expression (DEG) analysis of these clusters identified pericytes, SMC and four FB clusters (Supplementary Fig. 1B). These were PDGFR $\alpha^{\text {hi }}$ FB, two PDGFR $\alpha^{\text {lo }} C D 34^{\text {hi }}$ clusters that could be distinguished based on their expression of $C d 81$ (hereafter called $\mathrm{CD} 81^{+} \mathrm{FB}$ ) and $\operatorname{Igbp} 5$ (hereafter called $\operatorname{Igfbp} 5^{+} \mathrm{FB}$ ), and a PDGFR $\alpha^{\mathrm{lo}} \mathrm{CD} 34^{\mathrm{lo}}$ cluster that expressed higher levels of Fgfr2 (hereafter called Fgfr2 ${ }^{+}$FB) (Supplementary Fig. 1C). To determine how these clusters might relate to those identified in other, recently published scRNA-seq studies of small intestinal MSC ${ }^{7,11}$, DEGs from the previous MSC subsets were overlaid with our scRNA-seq dataset (Supplementary Fig. 1D). The MSC population termed "mural cells" by Hong et al ${ }^{11}$ corresponded to our pericytes, while their FB subsets termed $\mathrm{FB} 2,3,4$ and 5 corresponded to our small intestinal Igfbp5 ${ }^{+} \mathrm{FB}, \mathrm{Fgfr} 2^{+} \mathrm{FB}, \mathrm{CD} 81^{+} \mathrm{FB}$ and PDGFR $\alpha^{\text {hi }}$ FB clusters, respectively (Supplementary Fig. 1D). The signature genes of FB1 
114 identified by Hong et al as activated FB based on their expression of Junb and Fosb, were expressed widely by several MSC subsets in our dataset (Supplementary Fig. 1D), indicating

116 that this cluster represents a cell state rather than an MSC subset. Similar analysis of the MSC

117 datasets generated by McCarthy et $a l^{7}$ demonstrated that the PDGFR $\alpha^{\text {hi }}$ MSC subset they

118 defined as "telocytes" corresponded to our PDGFR $\alpha$ hi FB cluster, while their Lo-1 FB subset

119 corresponded to our CD81 ${ }^{+}$FB cluster and their Lo- 2 FB subset encompassed both our

$120 \mathrm{Fgfr}^{+}{ }^{+}$and Igfbp $5^{+}$FB clusters (Supplementary Fig. 1D) ${ }^{7}$. Thus, our results confirm and

121 extend recent findings and highlight the complexity of MSC subsets in the small intestinal

122 LP.

123 Louvain clustering also identified six MSC clusters in colon LP (Fig. 1B), which

124 DEG analysis identified as pericytes, SMC, and four FB clusters (Supplementary Fig. 1E).

125 These were PDGFR $\alpha^{\text {hi }} \mathrm{FB}$ and three PDGFR $\alpha^{\mathrm{lo}} \mathrm{CD} 34^{+}$clusters that could be distinguished

126 based on their expression of $C d 81$ (hereafter called CD81 $\mathrm{FB}$ ), $C D 90$ (hereafter called

$127 \mathrm{CD}^{+} 0^{+} \mathrm{FB}$ ) or Fgfr2 (hereafter called Fgfr2 ${ }^{+} \mathrm{FB}$ ) (Supplementary Fig. 1F). To determine the

128 relationship between the colonic and small intestinal MSC subsets, Pearson correlation

129 analysis was performed based on the pseudo-bulk of overlapping variable genes between the

130 two data sets. This showed that colonic pericytes, SMC, PDGFR $\alpha^{\text {hi }}$ FB, CD $81^{+}$FB closely

131 correlate with their counterparts in the small intestine, that colonic CD90 ${ }^{+} \mathrm{FB}$ most closely

132 correlate with small intestinal $\mathrm{Igfbp} 5^{+} \mathrm{FB}$ and that colonic Fgfr2 ${ }^{+} \mathrm{FB}$ closely correlate with

133 both Fgfr2 ${ }^{+}$and Igfbp5 $5^{+} \mathrm{FB}$ (Fig. 1C). 
Figure 1

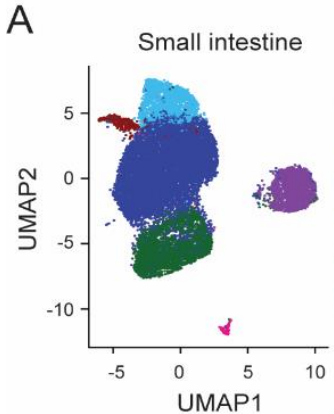

D

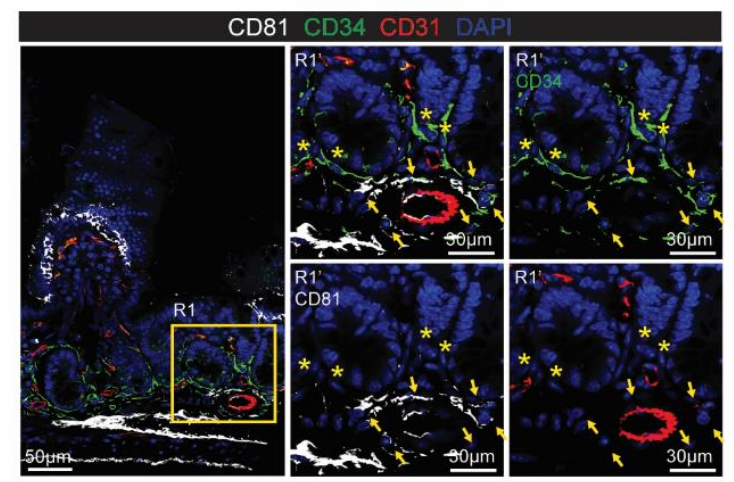

E
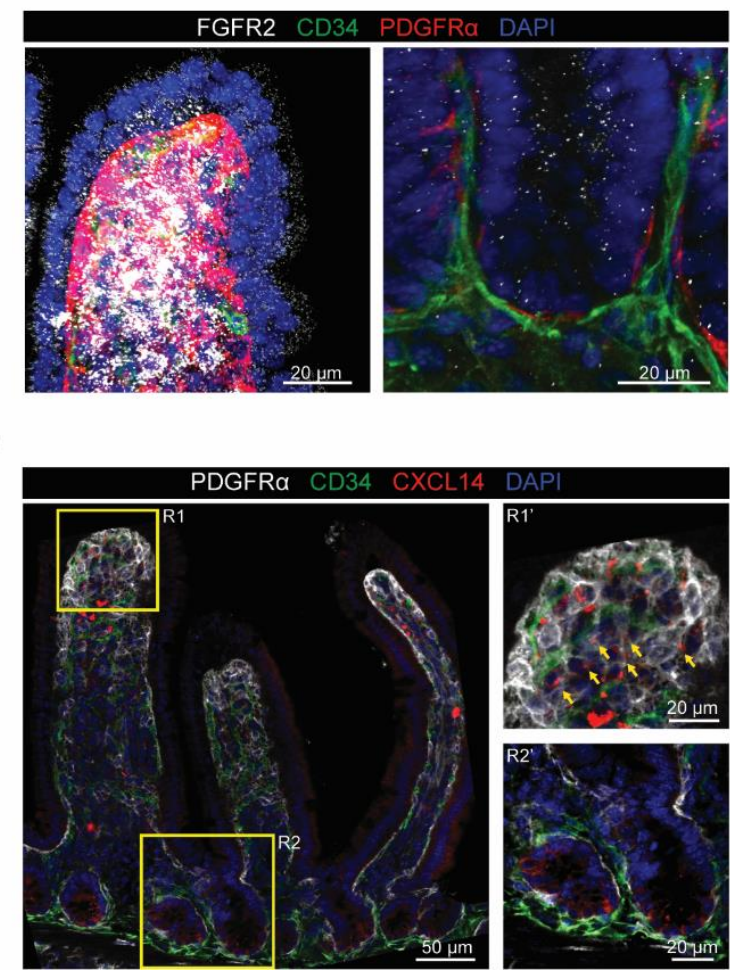

B

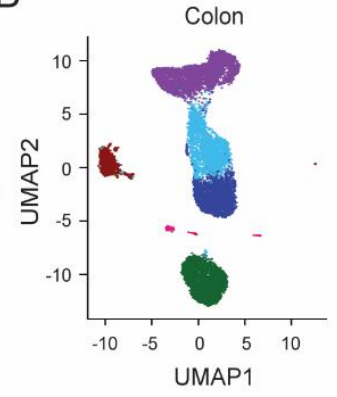

G

$\mathrm{H}$

।

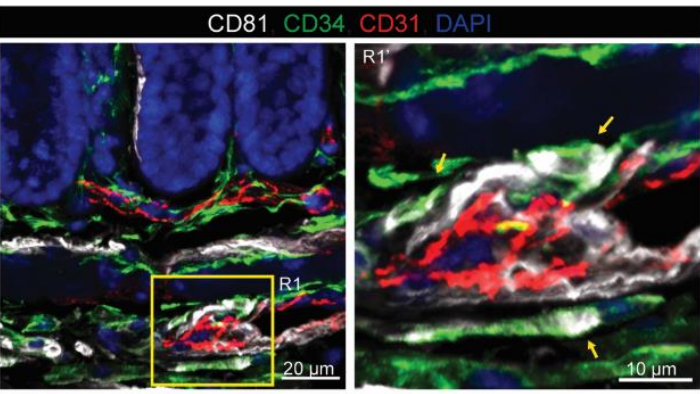

C
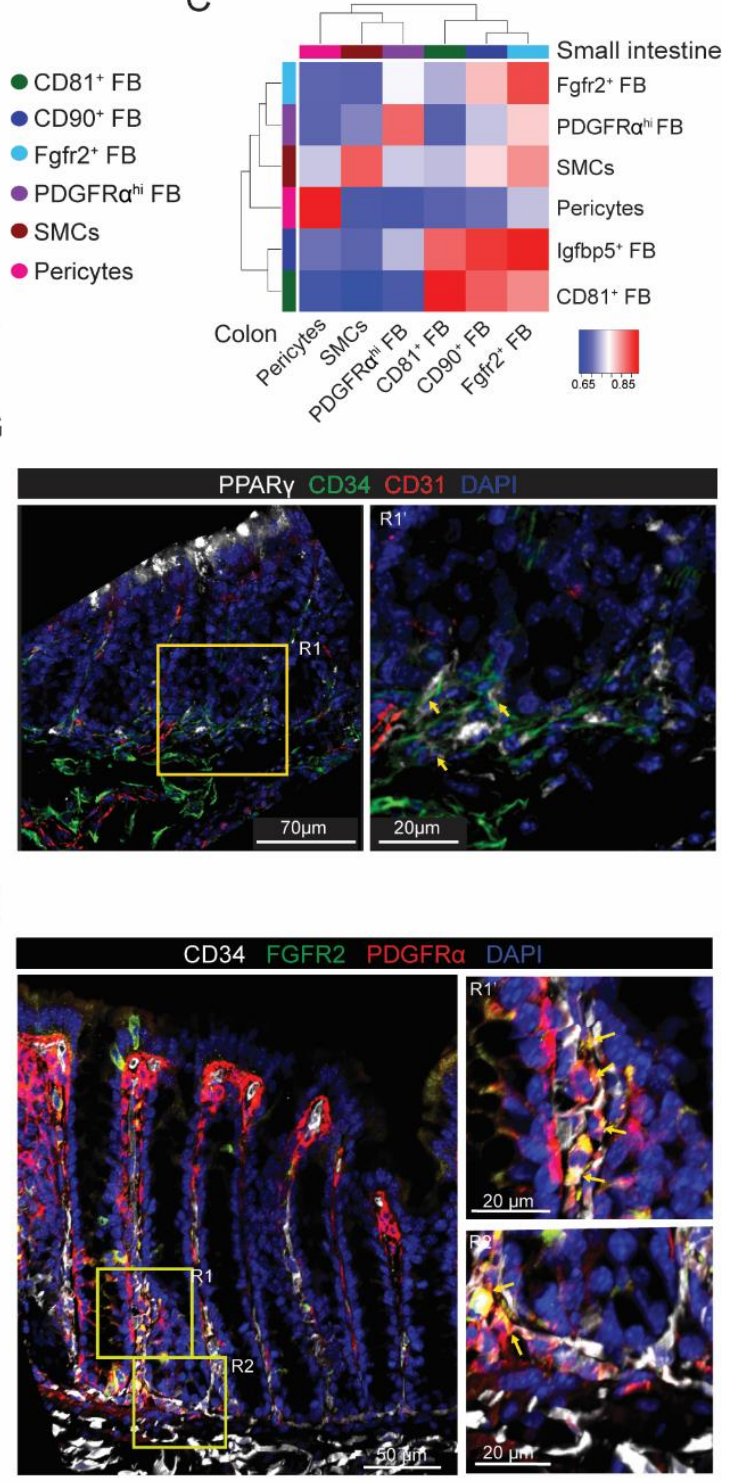

135 Figure 1. Intestinal MSC subsets are broadly conserved across intestinal segments. (A-

136 B) Uniform Manifold Approximation and Projection (UMAP) colored by unsupervised

137 Louvain clustering of murine small intestinal (A) and (B) colonic MSC. Results are from 2 
independent experiments/organ with 3 pooled mice/experiment. (C) Pearson correlations between averaged cluster expressions of Louvain clusters from small intestinal and colonic MSC based on 1301 overlapping variable genes. Unsupervised hierarchical clustering

141 indicate similarity of subsets within each tissue. (D-I) Immunohistochemical staining of

142 mouse jejunum (D-F) or colon (G-I) for indicated antigens. (D, F-I) Region (R)1' and R2' represent magnifications of R1 and R2 quadrants (yellow squares). (D) Arrows indicate location of $\mathrm{CD} 81^{+} \mathrm{FB}\left(\mathrm{CD} 81^{+} \mathrm{CD} 34^{+} \mathrm{CD} 31^{-}\right.$cells $)$and stars, location of Igfbp5 ${ }^{+} \mathrm{FB}(\mathrm{CD} 81-$ CD34+ CD31 cells). (E) Images of villus tip (left) and crypt (right). (F-I) Arrows indicate location of $(\mathbf{F}) \mathrm{Fgfr}^{+} \mathrm{FB}\left(\mathrm{CXCL} 14^{+} \mathrm{PDGFR} \alpha^{+} \mathrm{CD} 34^{-}\right.$cells), (G) $\mathrm{CD}^{-} 0^{+} \mathrm{FB}$ $\left(\mathrm{PPAR} \gamma^{+} \mathrm{CD} 34^{+} \mathrm{CD} 31^{-}\right.$cells $),(\mathbf{H})$ Fgfr2 ${ }^{+} \mathrm{FB}\left(\mathrm{Fgfr} 2^{+} \mathrm{CD} 34^{+} \mathrm{PDGFR}^{+}\right.$cells $)$and $(\mathbf{I}) \mathrm{CD} 1^{+}$ FB $\left(\mathrm{CD} 81^{+} \mathrm{CD} 34^{+} \mathrm{CD} 31^{-}\right.$cells $)$. Results are representative stains from $(\mathbf{D}-\mathbf{F}, \mathbf{H}) 2$ and $(\mathbf{G}$ and I) 3 experiments. See also Supplementary Fig. 1.

FB subsets are located in distinct niches along the crypt-villus axis

There is increasing evidence that subsets of small intestinal FBs may occupy distinct anatomical niches that overlap with the WNT/BMP signaling gradient along the crypt-villus axis ${ }^{7,10,30}$. In line with a recent report ${ }^{14}$, we found that small intestinal CD34+ $\mathrm{FB}$ (which include $\mathrm{CD} 81^{+}$and $\operatorname{Igfbp} 5^{+} \mathrm{FB}$ ) were located around crypts and in the submucosa, but were largely excluded from the villus core (Supplementary Fig. 1G). Of these, CD $34^{+} \mathrm{CD} 81^{+} \mathrm{FB}$ located around $\mathrm{CD} 31^{+}$vessels close to and within the submucosa, with some locating close to crypts (Fig. 1D), consistent with recent reports ${ }^{7,11,16}$, while $\mathrm{Igfbp}^{+}\left(\mathrm{CD} 34^{+} \mathrm{CD}^{-} 1^{-}\right) \mathrm{FB}$

159 located around crypts (Fig. 1D). Conversely, PDGFR $\alpha^{+}$CD $34^{-}$FB (including both PDGFR $\alpha^{\text {hi }}$ $160 \mathrm{FB}$ and PDGFR $\left.\alpha^{\mathrm{lo}} \mathrm{Fgfr} 2^{+} \mathrm{FB}\right)$ were located directly underlying the epithelium and within the villus core (Supplementary Fig. 1G). Of these, the Fgfr2 ${ }^{+}$FB were located within the villus core towards the tip of the villus (Fig. 1E); this was confirmed using CXCL14 as a marker for 
this subset (Supplementary Fig. 1H and Fig. 1F). In contrast, the PDGFR $\alpha^{\text {hi }}$ FB lay directly under the epithelium and at the villus tip (Fig. 1E, Fig. 1F, Supplementary Fig. 1G), supporting previous findings $\mathbf{s}^{7,9,11,30,31}$.

As in the small intestine, colonic $\mathrm{CD} 34^{+} \mathrm{FB}$ subsets located beneath and surrounding intestinal crypts, while PDGFR ${ }^{\text {hi }}$ FB formed a thin layer directly underlying the epithelium and were concentrated at the top of crypts (Supplementary Fig. 1I). Colonic CD $90^{+} \mathrm{CD} 34^{+}$ FB, which expressed high levels of Pparg (Supplementary Fig. 1H) could be identified after staining for PPAR $\gamma$ and were located at the base of colonic crypts (Fig. 1G). Colonic Fgfr2 ${ }^{+}$ FB localized preferentially between crypts (Fig. $1 \mathrm{H}$ ), whereas the colonic CD81 ${ }^{+}$FB located below the crypts and in the submucosa (Fig. 1I). Collectively these results demonstrate that the FB subsets identified by scRNA-seq locate within distinct niches of the small and large intestine.

\section{Expression of epithelial support genes is conserved across FB subsets in the small}

\section{intestine and colon}

Recent studies have suggested a division of labor amongst small intestinal FB subsets in the production of epithelial support factors ${ }^{5-9}$ and we thus assessed the expression of such genes in our small intestinal and colonic FB datasets. Consistent with previous studies ${ }^{7,10}$, small intestinal PDGFR $\alpha^{\text {hi }}$ FB were major producers of BMPs and this property was shared by colonic PDGFR $\alpha^{\text {hi }}$ FB (Fig. 2A). Bmp3, Bmp5 and Bmp7 expression was largely restricted to PDGFR $\alpha^{\text {hi }}$ FB, while expression of Bmpl, Bmp2 and Bmp4 was found more broadly among FB MSC subsets in both tissues (Fig. 2A). Both small intestinal and colonic PDGFR ${ }^{\text {hi }}$ FB were also the dominant source of the non-canonical WNT ligands, Wnt4, Wnt5a and Wnt5b, although $\mathrm{Fgfr}^{+} \mathrm{FB}$ also expressed Wnt4, particularly in the small intestine (Fig. 2A).

Consistent with previous results ${ }^{7,9}, \mathrm{CD} 81^{+} \mathrm{FB}$ were the major source of the BMP antagonist 
188 Greml in the small intestine and this was also highly expressed by colonic CD $81^{+} \mathrm{FB}$.

189 However, in the colon, Fgfr2 ${ }^{+}$and $\mathrm{CD} 90^{+} \mathrm{FB}$ also expressed Grem1 (Fig. 2A). Thus, the 190 specialization of MSC subsets in their expression of epithelial support genes is largely 191 conserved between the small intestine and colon. 
Figure 2

A

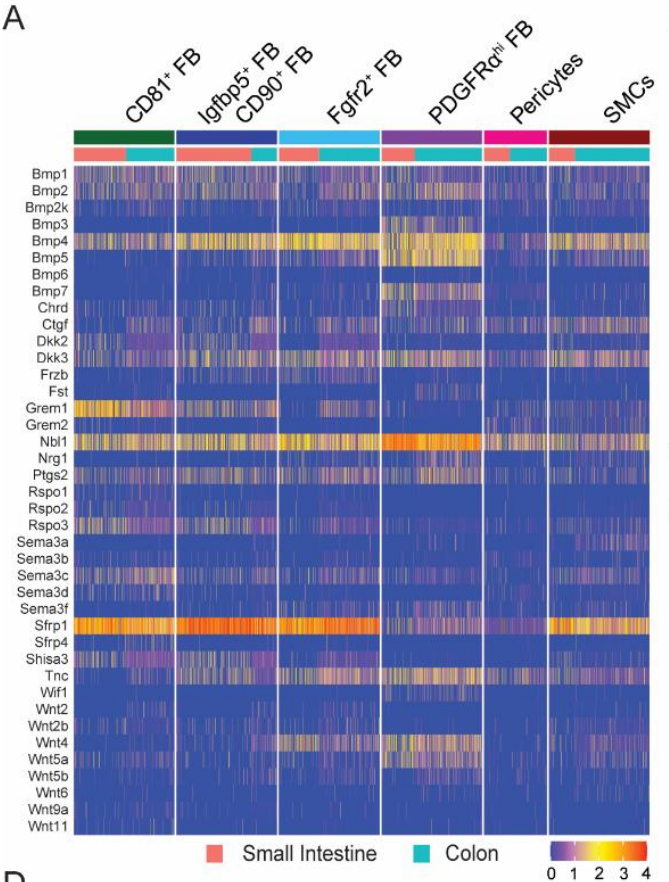

D $\mathrm{B}^{+} \mathrm{MSC}$

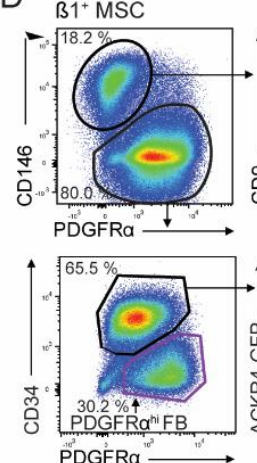

G

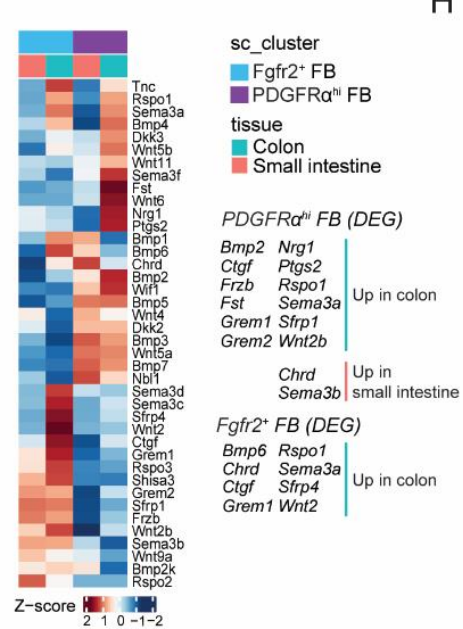

192

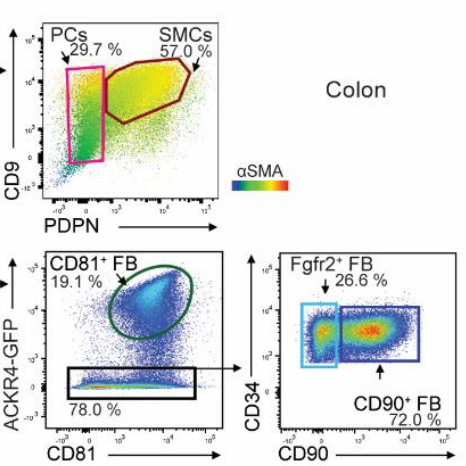

$\mathrm{H}$
B

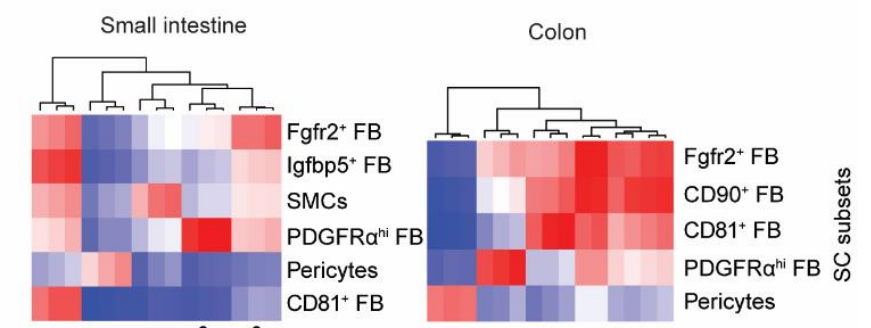

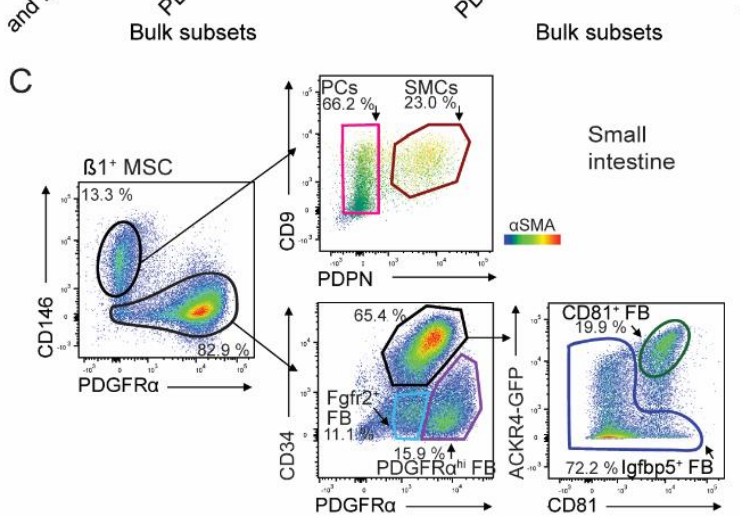

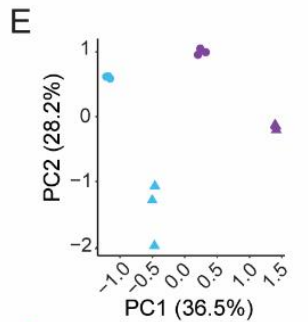

$$
\mathrm{F}
$$

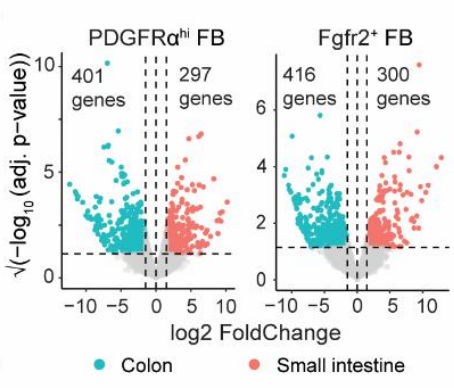

$\begin{array}{ll}\text { - Fgfr } 2^{+} \mathrm{FB} & \text { - Colon } \\ \text { - PDGFRa } & \text { PB }\end{array}$

- Small intestine
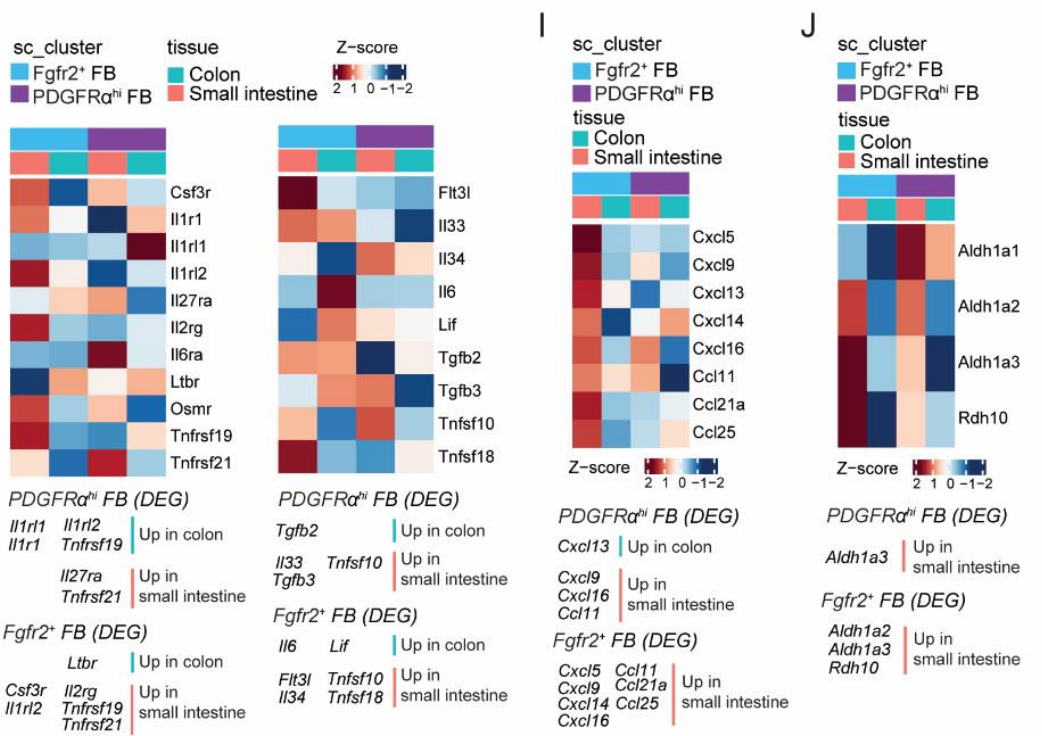

PDGFRa ${ }^{\text {hi }} F B$ (DEG)

Tgfb2 |Up in colon

11133 Tnfsf10 Up in

Fgfr2 ${ }^{+} F B$ (DEG)

116 Lif | Up in colon

Flt3l Tnfsf10 Up in

\begin{tabular}{ll|l} 
Flt31 & Tnfsf10 & Up in \\
II34 & Tnfsf18 & small intestine
\end{tabular}

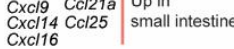

193 Figure 2. Despite similar FB subset composition, small intestinal and colonic FB display

194 regional transcriptional specialization. (A) Heatmaps showing scaled expression 
195 (integrated data) of selected epithelial support genes by indicated MSC subsets. (B) Pearson correlations between averaged cluster expressions of Louvain clusters from scRNA-seq and bulk RNA-seq datasets based on 1937 (small intestine, left) and 1925 (colon, right) overlapping variable genes. Bulk RNA-seq data is from sorted MSC subsets from 3 independent experiments. Unsupervised hierarchical clustering indicate similarities of bulk RNA-seq subsets within each tissue. (C-D) Flow cytometric analysis of adult small intestinal

(D) $\operatorname{Itg} \beta 1^{+}$MSCs from Ackr4.GFP mice. Representative staining of 2 pericytes; SMCs - smooth muscle cells, FB - fibroblast. (E) Principal component analysis (PCA) of bulk RNA-seq data from indicated sorted FB populations. Results are from 3 independent sorts/population. (F) Volcano plots showing differentially expressed genes (DEGs) between small intestinal and colonic PDGFR ${ }^{\text {hi }}$ FB (left) and Fgfr2+ FB (right). Dotted horizontal line denotes significant adjusted p-value of 0.05 , vertical dotted lines denote $\log _{2} \mathrm{FC}=0$ and the $\log _{2} \mathrm{FC}$ of $+/-1.5$. (G-J) Heatmap representations of averaged transcription levels of indicated genes within sorted FB subsets. Data are averaged from 3 independent bulk RNA-seq datasets. $(\mathbf{G})$ Epithelial support genes, $(\mathbf{H})$ cytokines and cytokine receptors, (I) chemokines, (J) vitamin A metabolism. Gene lists for I were selected based on the epithelial support list in (A) while those in $\mathbf{H}-\mathbf{J}$ were differentially expressed between either small intestinal and colonic PDGFR $\alpha^{\text {hi }}$ FB or between small intestinal and (H-J) the heat maps and. See also Supplementary Fig. 2.

\section{Small intestinal and colonic PDGFR $\alpha^{\text {hi }}$ FB and Fgfr2 ${ }^{+}$FB display regional}


219 To gain a broader understanding of how the major PDGFR $\alpha^{\text {hi }}$ and Fgfr2 ${ }^{+}$FB subsets in the small and large intestine might be related, we examined our scRNA-seq datasets for surface markers that would allow us to identify and sort these cells for bulk RNA-seq analysis

222 (Supplementary Fig. 2A). For the small intestine, pericytes were identified and sorted as 223 PDGFR $\alpha^{-}$ESAM- $1^{+} \mathrm{PDPN}^{-}$cells, $\mathrm{SMC}$ as PDGFR $\alpha$ ESAM- $1^{+} \mathrm{PDPN}^{+}$cells, $\mathrm{PDGFR} \alpha^{\mathrm{hi}} \mathrm{FB}$ as

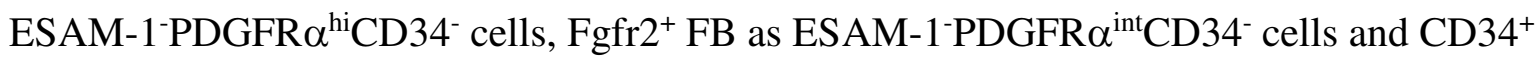
FB (including $\mathrm{CD} 81^{+}$and $\operatorname{Igfbp} 5^{+} \mathrm{FB}$ ) as $\mathrm{ESAM}-1^{-} \mathrm{PDGFR} \alpha^{\text {int }} \mathrm{CD} 34^{+}$cells. For the colon, pericytes were sorted as for small intestine, PDGFR $\alpha^{\text {hi }}$ FB was sorted as ESAM-1' $\mathrm{PDPN}^{+} \mathrm{CD} 34^{-}$cells, $\mathrm{Fgfr}{ }^{+} \mathrm{FB}$ were sorted as $\mathrm{ESAM}-1^{-} \mathrm{PDPN}^{\mathrm{hi}} \mathrm{CD} 34^{+} \mathrm{CD} 90^{-}$cells, CD90 ${ }^{+}$FB as $\mathrm{ESAM}-1^{-} \mathrm{CD} 34^{+} \mathrm{PDPN}^{\mathrm{hi}} \mathrm{CD} 90^{+}$cells and $\mathrm{CD} 81^{+} \mathrm{FB}$ as $\mathrm{ESAM}^{-} 1^{-} \mathrm{PDPN}{ }^{\text {int }} \mathrm{CD} 34^{+} \mathrm{CD} 90^{-}$ cells, based on the fact that colonic $\mathrm{CD} 81^{+} \mathrm{FB}$ express low levels of PDPN compared with the other $\mathrm{CD} 34^{+}$colonic FB subsets (Supplementary Fig. 2B). Correlation analysis of these bulk sorted intestinal FB subsets with the scRNA-seq data confirmed the accuracy of this staining strategy to identify small intestinal and colonic FB subsets by flow cytometry (Fig. 2B and Fig S2C). This initial panel was then refined for use in subsequent flow cytometry based analysis by including anti-CD81 to positively identify $\mathrm{CD} 81^{+} \mathrm{FB}$ directly, together with anti-CD146 (Fig. 2C and D), which can be used interchangeably with ESAM-1 (Supplementary Fig. 2D). CD81+ FB also expressed the atypical chemokine receptor, ACKR4, as assessed using Ackr4.GFP reporter mice (Fig. 2C and D) ${ }^{16}$, consistent with previous reports ${ }^{7,9,11,16}$ and our scRNA-seq analysis (Supplementary Fig. 1B).

240 from one another in PC1, while PC2 separated small intestinal from colonic FB (Fig. 2E),

241 suggesting that anatomical location has a major impact on the transcriptional profile of these

242 FB subsets. Consistent with this, small intestinal and colonic PDGFR $\alpha^{\text {hi }}$ FB differed in their 
transcription of 698 genes, while the two populations of $\mathrm{Fgfr} 2^{+} \mathrm{FB}$ differed in their

244 transcription of 716 genes (Fig. 2F, see Supplementary Table 1 (for PDGFR $\alpha^{\text {hi }}$ FB) and

245 Supplementary Table 2 (for Fgfr $2^{+}$FB) for complete list). Of these, 149 genes were

246 differentially expressed between the small intestine and colon in both FB subsets

247 (Supplementary Table 3); this included numerous Hox genes (Supplementary Fig. 2E),

248 consistent with the role of mesoderm in specifying the development of the different intestinal

249 segments ${ }^{32}$. Enrichr based analysis (Bioplanet $2019^{33}$ ) showed that most of the upregulated

250 pathways in the two subsets were in colon compared with small intestine, with few being

251 upregulated in small intestine compared with colon (Supplementary Fig. 2F). Irrespective of

252 their location, PDGFR $\alpha^{\text {hi }} \mathrm{FB}$ and $\mathrm{Fgfr}^{+} \mathrm{FB}$ showed very distinct expression of epithelial

253 support genes (Fig. 2G), suggesting these populations play discrete roles in maintaining the

254 epithelium; many of these genes were expressed at significantly higher levels in colonic subsets compared with their small intestinal counterparts (Fig. 2G). PDGFR $\alpha^{\text {hi }}$ FB and Fgfr $2^{+} \mathrm{FB}$ also expressed a wide range of immunologically relevant genes in both a subsetand tissue-specific manner (Fig. 2H-J). This included several cytokine and cytokine receptors (Fig. 2H), while small intestinal but not colon Fgfr $2^{+} \mathrm{FB}$ expressed a wide range of chemokines (Fig. 2I). Both subsets of small intestinal FB also expressed enzymes implicated in vitamin A metabolism, including the generation of retinoic acid (Fig. 2J), a major regulator of small intestinal immune responses. Collectively, these results highlight the unique functions of intestinal PDGFR $\alpha^{\text {hi }} \mathrm{FB}$ and $\mathrm{Fgfr}^{+} \mathrm{FB}$ and show that these vary depending on anatomical location.

\section{Intestinal precursors in E12.5 intestine can give rise to all adult intestinal MSC subsets}

266 While adult small intestinal and colonic LP contains multiple phenotypically,

267 transcriptionally, and spatially distinct MSC subsets, the developmental relationship between 
these subsets and whether all derive from similar precursors remains unclear ${ }^{17,27,28}$. To explore this, we first investigated which MSC might be present in the small intestine and colon of E12.5 embryos by flow cytometry (Fig. 3A and S3A). In contrast to adult mice (Fig. $2 \mathrm{C}$ and D), E12.5 small intestinal and colonic $\operatorname{Itg} \beta 1^{+}$cells consisted of one major population of PDGFR $\alpha^{+} \mathrm{CD} 34^{-}$MSCs, together with a small subset of PDGFR $\alpha^{-}$cells that expressed the mesothelial markers dipeptidyl peptidase-4 (DPP4, CD26) and PDPN (Fig. 3A and B) ${ }^{34}$. To assess whether these populations could give rise to the MSC subsets found in the adult intestine, small and large intestine were dissected from E12.5 embryos and transplanted under the kidney capsule of adult WT recipient mice (Fig. 3C). Embryonic intestines from mice ubiquitously expressing EYFP were used for these experiments in order to trace the development of donor derived $\left(\mathrm{EYFP}^{+}\right) \mathrm{MSC}$ within grafted tissues. As expected ${ }^{35-37}$, small intestinal and colonic grafts had increased markedly in size by 4-6 weeks post transplantation (Fig. 3C) and contained mucosa that histologically resembled that of adult small intestine and colon, respectively (Supplementary Fig. 3B). To assess the phenotypic diversity of graftderived MSC, small intestinal and colonic grafts were isolated 4 weeks after transplantation, digested, and the expression of MSC subset markers on embryonically derived (YFP $\left.{ }^{+}\right) \operatorname{Itg} \beta 1^{+}$ MSC assessed by flow cytometry (Fig. 3D, Fig S3C). Both small intestinal and colonic grafts contained putative populations of graft-derived SMC $\left(\mathrm{CD} 146^{+} \mathrm{PDGFR}^{-} \mathrm{PDPN}^{+}\right)$, pericytes $\left(\mathrm{CD} 146^{+} \mathrm{PDGFR}^{-} \mathrm{PDPN}^{-}\right), \mathrm{PDGFR} \alpha^{\mathrm{hi}} \mathrm{FB}\left(\mathrm{CD} 146^{-\mathrm{CD}} 34^{-/ / \mathrm{lo}} \mathrm{PDGFR} \alpha^{\mathrm{hi}}\right), \mathrm{CD} 81^{+} \mathrm{FB}\left(\mathrm{CD} 146^{-}\right.$ PDGFR $\left.\alpha^{\text {lo }} \mathrm{CD}^{2} 1^{+} \mathrm{CD} 34^{+}\right)$and $\mathrm{CD} 81^{-} \mathrm{CD} 34^{+} \mathrm{FB}\left(\mathrm{CD} 146^{-} \mathrm{PDGFR}^{\mathrm{lo}} \mathrm{CD} 34^{+} \mathrm{CD} 81^{-}\right)$(Fig. 3D). To confirm the presence of these MSC subsets in the grafts, $\mathrm{YFP}^{+} \operatorname{Itg} \beta 1^{+} \mathrm{MSC}$ were sorted from grafted colon and subjected to scRNA-seq (Supplementary Fig. 3C). UMAP dimensionality reduction and Louvain clustering identified eight clusters (Fig. 3E), two of which (clusters 6 and 7) were identified as ICC and mesothelial cells, respectively ${ }^{38-40}$ (Supplementary Fig. 3D). These clusters were not part of our adult MSC datasets, as ICC 
293 were removed bioinformatically and the mesothelium was removed together with the

294 muscularis externa during tissue processing. Pearson correlation analysis based on the

295 pseudobulk of overlapping variable genes identified cluster 3 as being similar to adult

296 PDGFR $\alpha^{\text {hi }}$ FB, cluster 4 as pericytes and cluster 5 as SMC (Fig. 3E and F). The remaining

297 three clusters (clusters 0-2) were more closely related to the three adult CD $34^{+}$FB subsets,

298 with cluster 1 most closely correlated to $\mathrm{CD} 81^{+} \mathrm{FB}$, cluster 0 most closely correlated to

$299 \mathrm{Fgfr} 2^{+} / \mathrm{CD} 90^{+} \mathrm{FB}$ and cluster 2 showing equivalent correlation to all three adult $\mathrm{CD} 34^{+} \mathrm{FB}$

300 subsets (Fig. 3F). Furthermore, the distinct expression of epithelial support genes by each of

301 the four FB subsets largely overlapped with the pattern seen in adult intestine (Fig. 3G, Fig.

302 2A). Collectively, these results suggest that embryonal MSC precursors present in E12.5

303 intestine can give rise to all adult intestinal MSC subsets. 
A

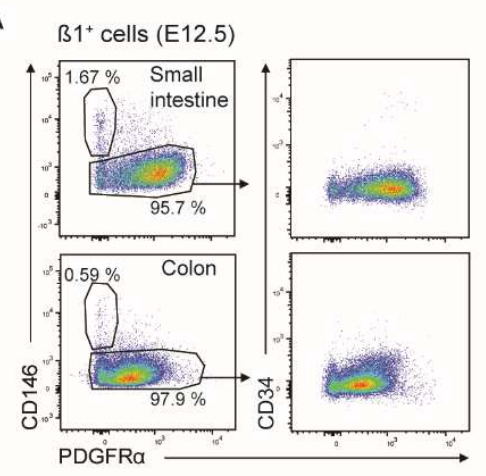

D
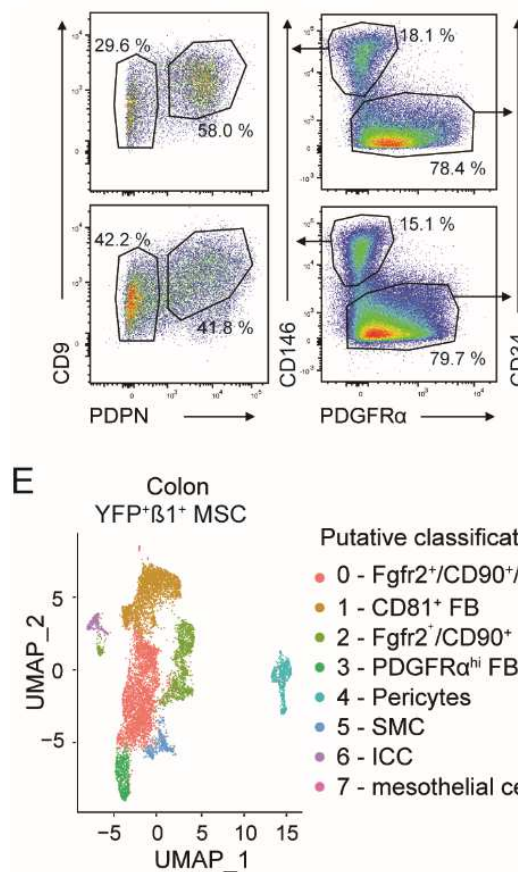

Putative classificatio - 1 - CD81+FB

- 2 - Fgfr ${ }^{+} / \mathrm{CD} 90^{+} \mathrm{FB}$

- 3 - PDGFRa ${ }^{\text {hi }}$ FB

- 4 - Pericytes

- 5 - SMC

- 6 - ICC

- 7 - mesothelial cells
B

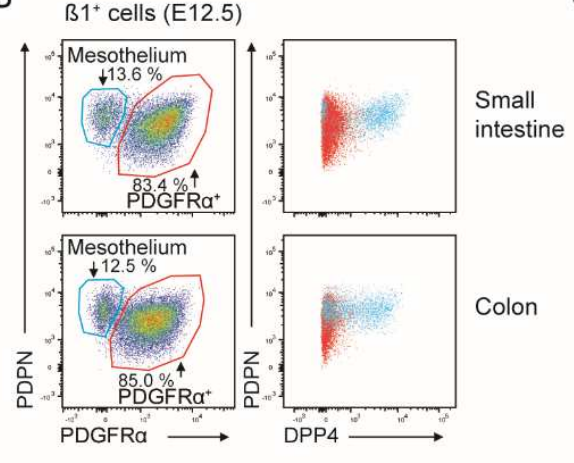

C

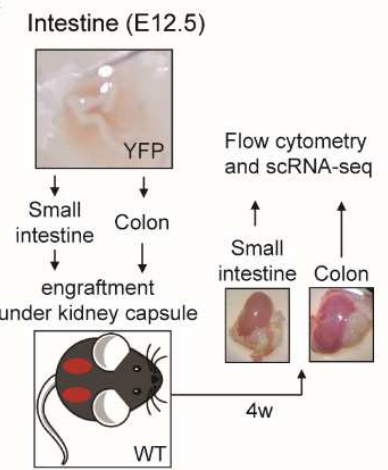

G
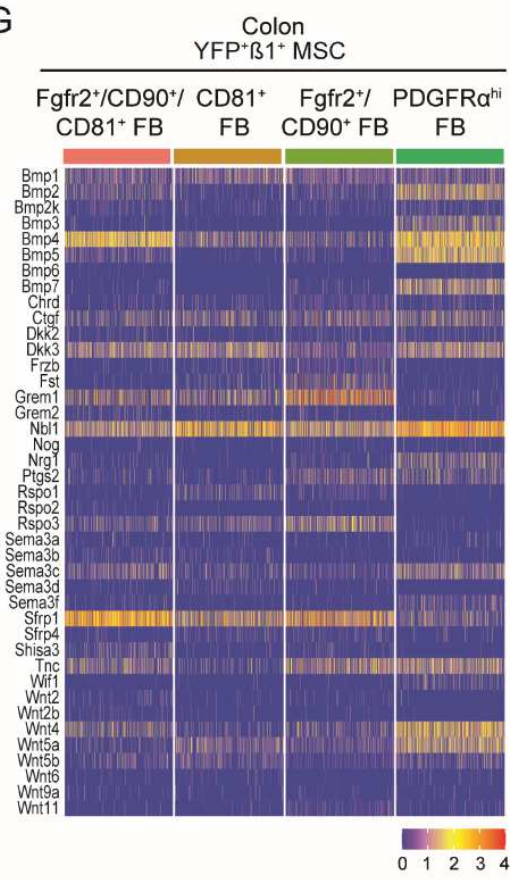
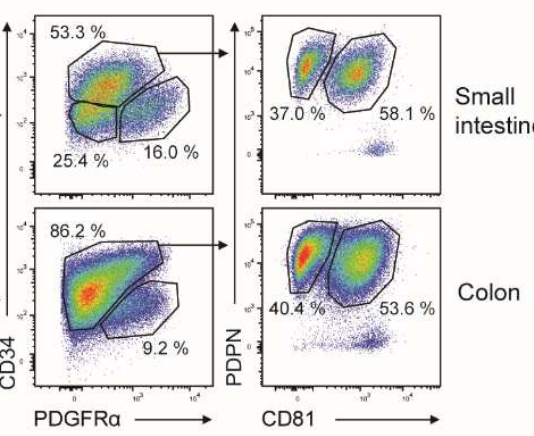

\section{F} Colon - 0 - $\mathrm{Fgfr}^{+} / \mathrm{CD} 90^{+} / \mathrm{CD} 81^{+} \mathrm{FB}$

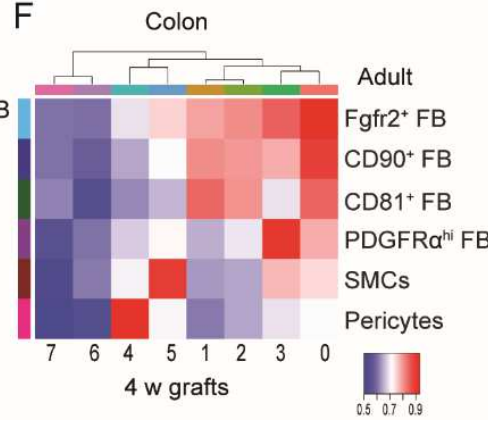

Fgfr2 ${ }^{+}$FB $\mathrm{CD} 90^{+} \mathrm{FB}$ $\mathrm{CD} 81^{+} \mathrm{FB}$ DGFRa ${ }^{\text {hi }} F B$ SMCs

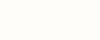

Figure 3. Adult intestinal MSC subsets derive from intestinal precursors present in

E12.5 intestine. (A-B) Flow cytometric analysis of Itg $\beta 1^{+}$MSCs isolated from indicated

organs on embryonic day (E) 12.5. (B) Right hand plots show expression of DPP4 (CD26) on gated PDPN ${ }^{+}$PDGFR $\alpha^{-}$(blue) and PDPN ${ }^{+}$PDGFR $^{+}$(red) cells from plots on left. Data are representative of (A) 4 experiments with 2-8 embryos/experiment, or (B) 3 experiments with

310 6-8 individual embryos. (C) Workflow of transplantation of E12.5 intestine from $\mathrm{YFP}^{+}$mice

311 under the kidney capsule of WT recipients. (D) Flow cytometric analysis of YFP ${ }^{+} \operatorname{Itg} \beta 1^{+}$

312 MSC in intestinal grafts 4 weeks after transplantation. Results are representative of 2 

from 8624 single cells from 3 pooled colonic grafts with an average of 2223 genes/cell. (F)

317 Pearson correlations of averaged gene expression in colonic graft and adult colon MSC clusters based on 1486 overlapping variable genes. (G) Heatmap showing scaled transcription levels (integrated data) of selected epithelial support genes within the putative corresponding FB clusters identified in (E). See also Supplementary Fig. 3.

\section{Adult intestinal MSC derive from $\mathrm{Glil}^{+}$embryonic precursors}

323 To explore further the origin of adult MSC, we next lineage-traced E12.5 MSC and mesothelium into adulthood. GLI1 is a transcription factor induced by active hedgehogsignaling and is expressed by MSC in multiple organs ${ }^{41}$. PDGFR $\alpha^{+} \mathrm{CD} 34^{-} \mathrm{MSC}$ and PDGFR $\alpha{ }^{-} \mathrm{PDPN}^{+}$mesothelial cells from the small intestine and colon of E12.5 Glil-EGFP embryos both expressed EGFP, whereas intestinal epithelial, endothelial and CD $45^{+}$cells did not (Fig. 4A and B). To lineage trace Glil-expressing cells into adulthood, female R26R.EYFP mice ${ }^{42}$ were mated with Glil-Cre.ERT2 males expressing the estrogen receptor (ERT2) under control of Gli1-Cre, and pregnant dams injected i.p with 4-hydroxytamoxifen (4-OHT) at E11.5 (Fig. 4C). Two days later, YFP expression had been induced in a small but consistent proportion of $\operatorname{Itg} \beta 1^{+} \mathrm{PDGFR} \alpha^{+} \mathrm{MSC}$ and mesothelial cells in the small intestine and colon of Gli1-CreERT2 ${ }^{+/-}$. R26R.EYFP embryos, but not in $\mathrm{Cre}^{-}$embryos (Supplementary Fig. 4A and B). Labeling was not observed in intestinal epithelial, endothelial, or CD45 ${ }^{+}$ immune cells of $G l i 1-C r e E R T 2^{+/-} . R 26 R$.EYFP mice and thus was specific to intestinal MSC and mesothelial cells (Supplementary Fig. 4A and B). 5-7 weeks after birth, similar proportions of YFP-expressing cells were detected in all mature MSC subsets in both the 
small intestine and colon (Fig. 4D). Collectively, these results demonstrate that $\mathrm{Glil}^{+}$cells present in the E12.5 intestine contain cells that give rise to all major adult intestinal MSC subsets.

Figure 4

A

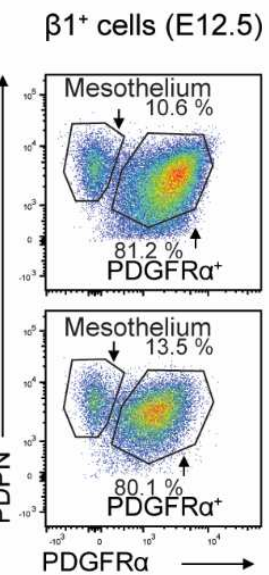

Gli1-EGFP
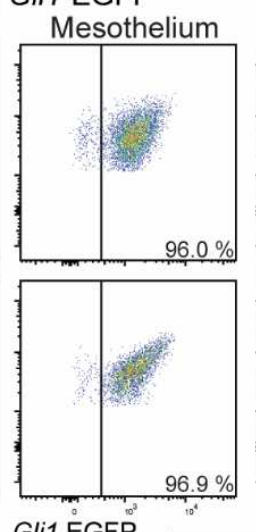

B E12.5 Gli1-EGFP
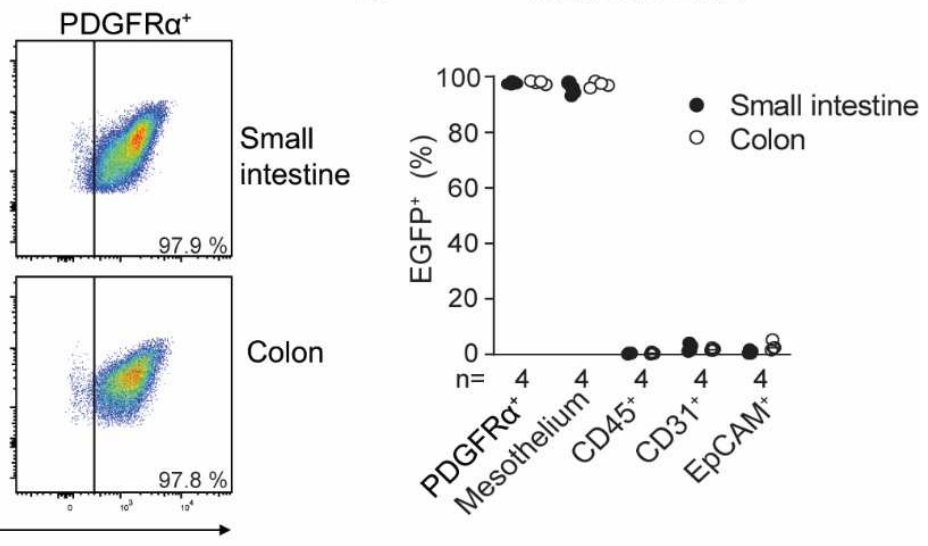

C

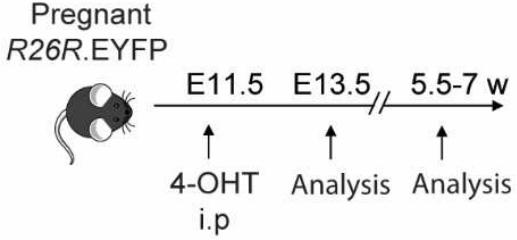

Pregnant

6R.EYFP

341

342

343

344

345

D

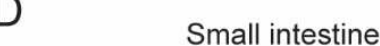

Colon

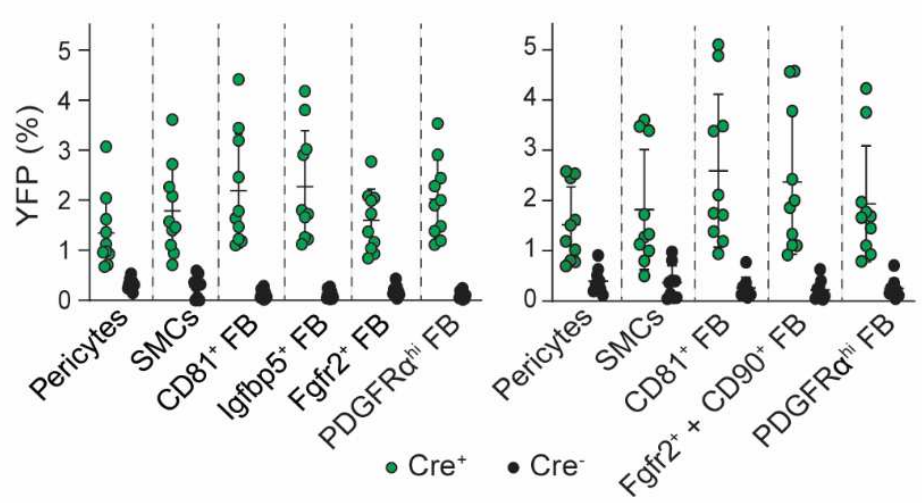

Figure 4. Adult intestinal MSC derive from Gli1 $^{+}$embryonic precursors.

(A) Representative flow cytometric analysis and (B) Proportions of indicated cells expressing EGFP in the small intestine and colon of embryonic E12.5 Gli1-EGFP mice. Results are from 8 individual embryos, with each circle representing an individual embryo. (C) Workflow of lineage-tracing experiments. R26R.EYFP females were mated overnight with Glil.CreERT2 $^{+/-}$males and pregnant dams injected i.p. with 4-Hydroxytamoxifen (4-OHT) at E11.5. (D) Proportions of indicated MSC subset expressing YFP in small intestine and colon 
of 5.5-7 week old Gli1.CreERT2 ${ }^{+/-} \cdot R 26 R$.EYFP and Gli1.CreERT2-/-R26R.EYFP littermates.

350 Results are from 4 independent experiments with 2-8 mice/experiment. Each circle represents an individual mouse. Bars represent the means and SD. See also Supplementary Fig. 4.

Trajectory analysis indicates that adult intestinal MSC subsets originate from embryonic Gli1 $^{+}$mesothelial cells

To gain further insights into the relationship between embryonic intestinal $\mathrm{Glil}^{+}$cells and adult intestinal MSC subsets, scRNA-seq was performed on fluorescently activated cell sorted $\operatorname{Itg} \beta 1^{+}$MSC from the colon of E12.5 embryos. Louvain clustering identified six clusters (Fig. 5A), one of which, cluster 4, was identified as mesothelial cells due to its expression of mesothelial associated markers ${ }^{39,40}$ (Fig. 5B). Consistent with our flow cytometric analysis (Fig. 3B), this cluster expressed transcripts for DPP4 and PDPN, but lacked expression of PDGFR $\alpha$ (Supplementary Fig. 5A). To determine the relationship between embryonic and adult MSC subsets, the embryonic and adult colonic datasets were integrated and tSPACE ${ }^{43}$ trajectory analysis was performed on MAGIC imputed sets of variable genes, as described previously ${ }^{44,45}$. Pericytes were removed from this analysis, as too few of these cells were present in the adult dataset to generate meaningful conclusions. Three-dimensional visualization of tSPACE principal components (tPC) 1-3 demonstrated that embryonic cells broadly clustered together and away from adult MSC subsets (Fig. 5C).

368 Nevertheless, two clear connections were observed between embryonic and adult colonic MSC (Fig. 5C, arrow heads). The first was a direct and distinct connection between embryonic clusters 0 and 2 and adult SMC, while the second was a connection between

371 embryonic clusters 4 (mesothelial cells) and 5 to adult $\mathrm{CD} 81^{+} \mathrm{FB}$ and to a lesser extent adult

$372 \mathrm{CD}^{+} \mathrm{FB}$ (Fig. 5C). Supporting the idea that the mesothelium gives rise to SMC and some

373 FB in the intestinal serosa and muscle layers of the intestine ${ }^{27,28}$, we found that both 
mesothelial cells and cluster 5 expressed several genes previously associated with FB progenitors ${ }^{46-51}$ (Supplementary Fig. 5B). We thus selected mesothelial cells as a tSPACE trajectory starting point for pseudotime analysis (Fig. 5D). This demonstrated a pseudotime trajectory of mesothelial cells to adult SMC via embryonic clusters 0 and 2 and from mesothelial cells and cluster 5 to adult $\mathrm{CD} 81^{+} \mathrm{FB}$ (Fig. 5D, arrows).

Interestingly, rather than branching immediately into distinct FB subsets, adult CD $81^{+}$ FB connected directly to $\mathrm{CD} 90^{+} \mathrm{FB}$ that then connected to $\mathrm{Fgfr}^{+} \mathrm{FB}$ and finally to PDGFR $\alpha^{\text {hi }}$ FB (Fig 5C), and tSPACE analysis of adult FB showed a similar linear connection between FB subsets (Fig. 5E). As adventitial CD81 ${ }^{+}$FB have been suggested to contain FB precursors in adults ${ }^{52}$, we used them as the starting population for a new pseudotime analysis, which again indicated a linear trajectory from adult $\mathrm{CD} 81^{+} \mathrm{FB}$ via $\mathrm{CD} 90^{+} \mathrm{FB}$ and Fgfr2 $^{+}$FB to PDGFR $\alpha^{\text {hi }}$ FB (Fig. 5F). This conclusion was further supported when we overlaid our trajectory on to the DEG genes for clusters generated by a recent pseudotime analysis of mouse tissue FB, which has suggested a developmental trajectory from $\mathrm{PilO}^{+}$ precursors through a population of $\operatorname{Col} 15 \alpha 1^{+} \mathrm{FB}$ that eventually gives rise to mature tissue specific FB that include $\mathrm{Fbln} \mathrm{I}^{+}$and then $\mathrm{Bmp4^{+ }} \mathrm{FB}$ in the intestine ${ }^{52}$. This analysis showed that the $P i 16^{+}, C o l 15 \alpha 1^{+}, F b \ln 1^{+}$and $B m p 4^{+} \mathrm{FB}$ clusters defined by Buechler et al broadly overlapped with our colonic $\mathrm{CD} 81^{+}, \mathrm{CD} 90^{+}, \mathrm{Fgfr} 2^{+}$and $\mathrm{PDGFR} \alpha^{\text {hi }} \mathrm{FB}$ subsets, respectively (Supplementary Fig. 5C). Collectively, these results suggest that adult MSC subsets originate from the embryonic $\mathrm{Gli}^{+}$mesothelium, with adult SMC deriving from an embryonic intermediate distinct from that which gives rise to adult FB. In addition, these results suggest that adult $\mathrm{FB}$ subsets arise sequentially from $\mathrm{CD} 81^{+} \mathrm{FB}$.

Each FB cluster had an extended appearance in tSPACE, with groups of cells streaming outwards from a central core (Fig 5E). To determine what processes might underlie this appearance, we assessed differences in gene expression between the start (core) and end 
399 (tip) of each FB cluster (Supplementary Fig. 5D). While cells at the tip and core of the 400 clusters had similar read counts and detected genes (Supplementary Fig. 5E), those at the 401 core of each subset expressed 108-121 genes at significantly higher levels than those at the

402 tip, while tip cells expressed no or few (0-2) genes at a significantly higher level. Enrichr 403 based analysis (GO biological process $2018^{53}$ ) of the genes expressed preferentially by cells 404 at the core demonstrated that 9 of the top 10 pathways were shared across FB subsets 405 (Supplementary Fig. 5F). These included processes involved in positive regulation of 406 transcription, responses to cytokines, and responses to unfolded proteins, with the 407 overwhelming majority of these genes being shared by the core cells in all the FB subsets 408 (Supplementary Fig. 5G). Together these results suggest that the tip cells within each FB 409 subset are more quiescent than their core counterparts and hence may be more highly 410 differentiated. 
A

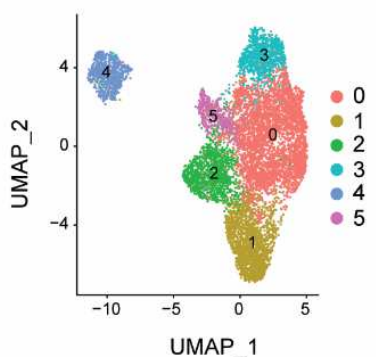

B

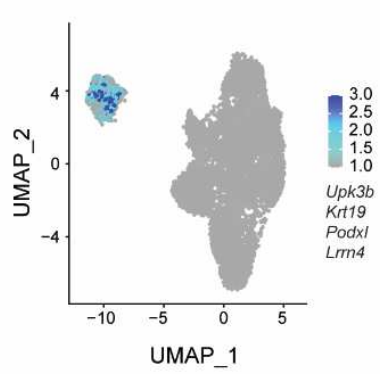

C

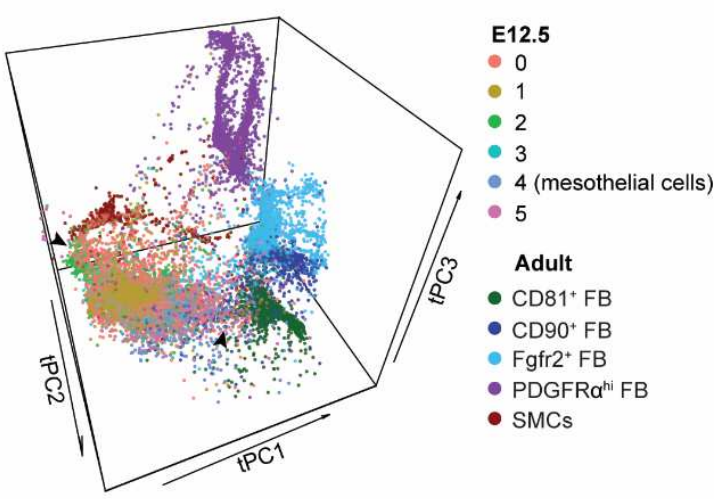

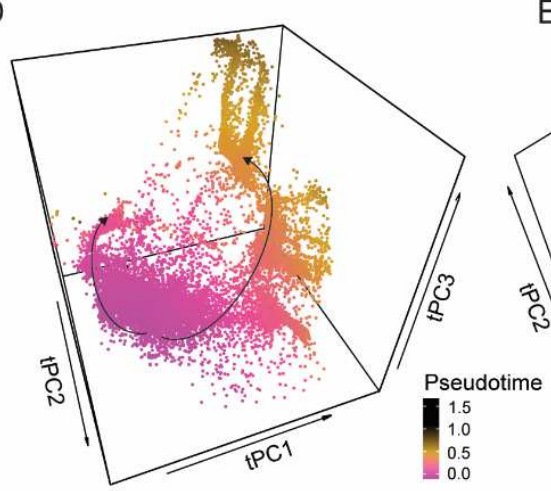

E

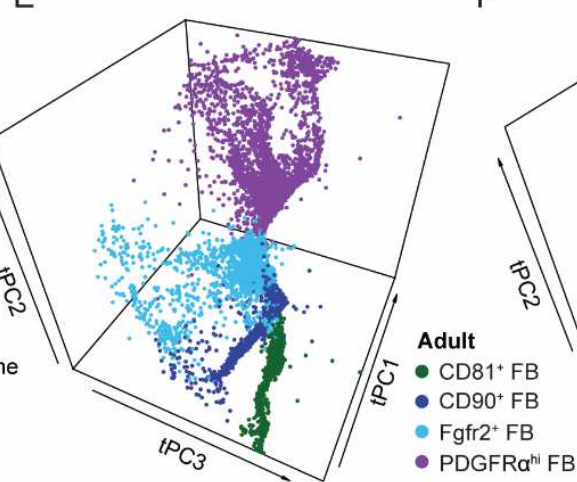

F

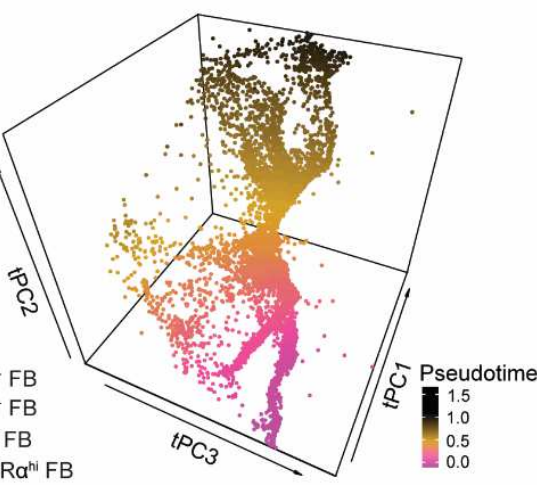

412

413

414

415

416

417

418

419

420

421

422

423

424

Figure 5. Trajectory analysis indicates that adult intestinal MSC subsets derive from

embryonic Gli1 $^{+}$mesothelial cells. (A) UMAP dimensionality reduction of scRNA-seq data colored by Louvain clustering from FACS purified $\operatorname{Itg} \beta 1^{+}$MSC from the colon of embryonic day E12.5 mice. Data are from 9632 single cells from 2 pooled experiments using 3-5

embryonic colons/experiment, with an average of 2521 genes/cell. (B) UMAP of E12.5 large intestinal Itg $\beta 1^{+}$MSC overlaid with expression of the indicated mesothelium associated genes. (C and D) tSPACE principal component analysis (tPC 1-3) projection of pooled adult colonic and E12.5 large intestinal MSC. (C) Clusters are color coded as in (A) for embryonic clusters or as in Fig. 1B for adult clusters. Arrow heads indicate connections between embryonic and adult clusters. (D) Pseudotime analysis using averaged values of the 9 trajectories with starting point in mesothelial cells superimposed on tPC 1-3. (E and F) tSPACE projections of adult colonic MSC in tPC1-3. (F) Pseudotime analysis superimposed 
426 Supplementary Fig. 5.

427

428 Colonic PDGFRa ${ }^{\text {hi }}$ FB consist of three transcriptionally distinct clusters originating 429 from Fgfr2 ${ }^{+}$FB.

430 tSPACE analysis of adult FB subsets indicated that PDGFR $\alpha^{\text {hi }}$ FB originated from Fgfr2 ${ }^{+}$FB and then separated into three branches (Fig. 5E and F). To validate the idea that Fgfr2 ${ }^{+} \mathrm{FB}$ act as precursors of PDGFR $\alpha$ hi FB we performed RNA velocity analysis ${ }^{54}$ focusing on these subsets, which confirmed the directionality from Fgfr2 ${ }^{+}$FB to PDGFR $\alpha^{\text {hi }}$ FB (Fig. 6A). Reclustering of only PDGFR $\alpha^{\text {hi }}$ FB uncovered three clusters that diverged along the three trajectory branches (Fig. 6B) that could be distinguished based on expression of $C d 9$ and 436 Cd141 (thrombomodulin (Thbd)) (Fig. 6C). This generated clusters of CD9hi CD141', $437 \mathrm{CD} 9{ }^{10} \mathrm{CD} 141^{+}$and $\mathrm{CD} 141^{\text {int }}$ cells, all of which expressed the "telocyte" marker, Foxll 438 (Supplementary Fig. 6A) ${ }^{10}$. Consistent with these findings, flow cytometric analysis of colonic PDGFR $\alpha^{\text {hi }}$ FB identified distinct clusters of CD $9{ }^{\text {hi }} \mathrm{CD} 141^{-}$and $\mathrm{CD} 9{ }^{\mathrm{lo}} \mathrm{CD} 141^{+}$cells, 440 together with CD9- cells that expressed heterogeneous levels of CD141 and which we 441 referred to as CD141 ${ }^{\text {int }}$ FB (Fig. 6D). Analysis of the top DEG between these populations demonstrated that CD9hi $\mathrm{CD} 141^{-}$cells expressed the highest levels of $\mathrm{Nrg} 1, \mathrm{Fgf7}$, Il1 $r l 1$ (ST2

443 (IL33 receptor)) and Ptgs 2 , that $\mathrm{CD} 9{ }^{\mathrm{lo}} \mathrm{CD} 141^{+}$cells expressed high levels of fibrosisassociated Aspn (Asporin), Il11ral and Cxcl12, while CD141 ${ }^{\text {int }}$ cells expressed high levels of

445 Cxcl10, Ly6c1, Adamdec1, Wnt4a and Plpp3 (Supplementary Fig. 6B). The CD9 ${ }^{\text {lo }}$ CD141 ${ }^{+}$ 446 cells, and to a lesser extent the CD $141^{\text {int }}$ cells, expressed mRNA and protein for $\alpha$ SMA 447 (Supplementary Fig. 6C), a marker of myofibroblasts but not "telocytes".

448 Immunohistochemical staining for PDGFR $\alpha$ and $\alpha \mathrm{SMA}$ showed that $\alpha \mathrm{SMA}^{+} \mathrm{PDGFR} \alpha^{\text {hi }}$ cells 449 localize preferentially to the isthmus area just above colonic crypts, while $\alpha$ SMA-PDGFR $\alpha^{\text {hi }}$ 
450 cells aligned directly underneath the epithelium at the top and bottom of crypts (Fig. 6F). The

$451 \mathrm{CD}^{\mathrm{lo}} \mathrm{CD} 141^{+}, \mathrm{CD} 9{ }^{\text {hi }} \mathrm{CD} 141^{-}$and $\mathrm{CD} 141^{\text {int }} \mathrm{FB}$ also differentially expressed several epithelial

452 support genes (Fig. 6G), suggesting that these populations may play distinct roles in

453 supporting the epithelium at different stages of its development. Thus, adult colonic

454 subepithelial PDGFR $\alpha^{\text {hi }}$ FB consist of spatially and transcriptionally distinct clusters that

455 derive from $\mathrm{Fgfr}^{+} \mathrm{FB}$.

Figure 6

A

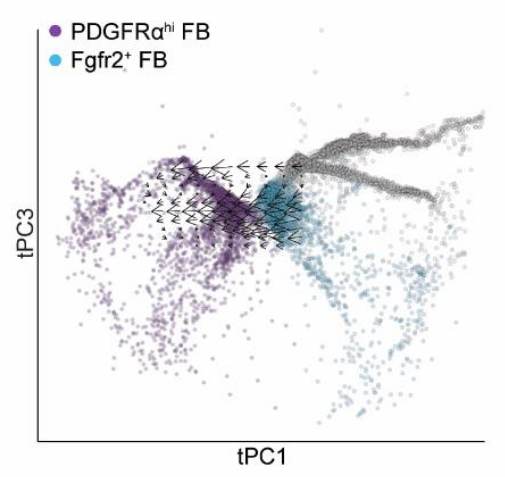

D

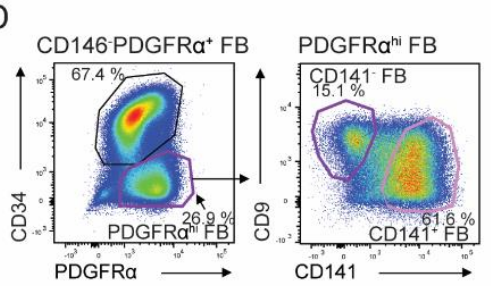

$\mathrm{F}$

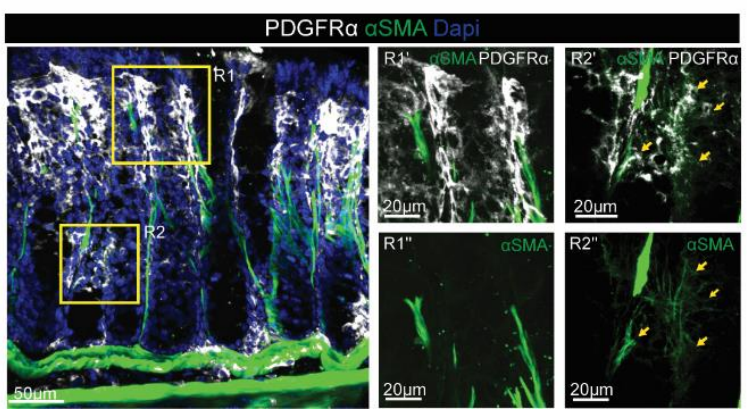

B

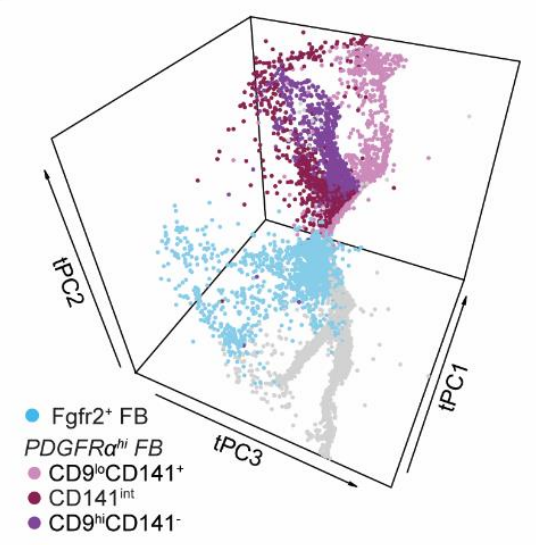

E Acta2 (aSMA)

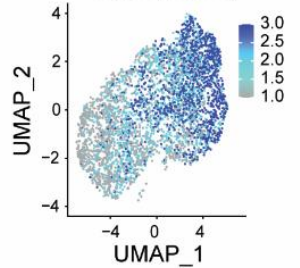

G

C
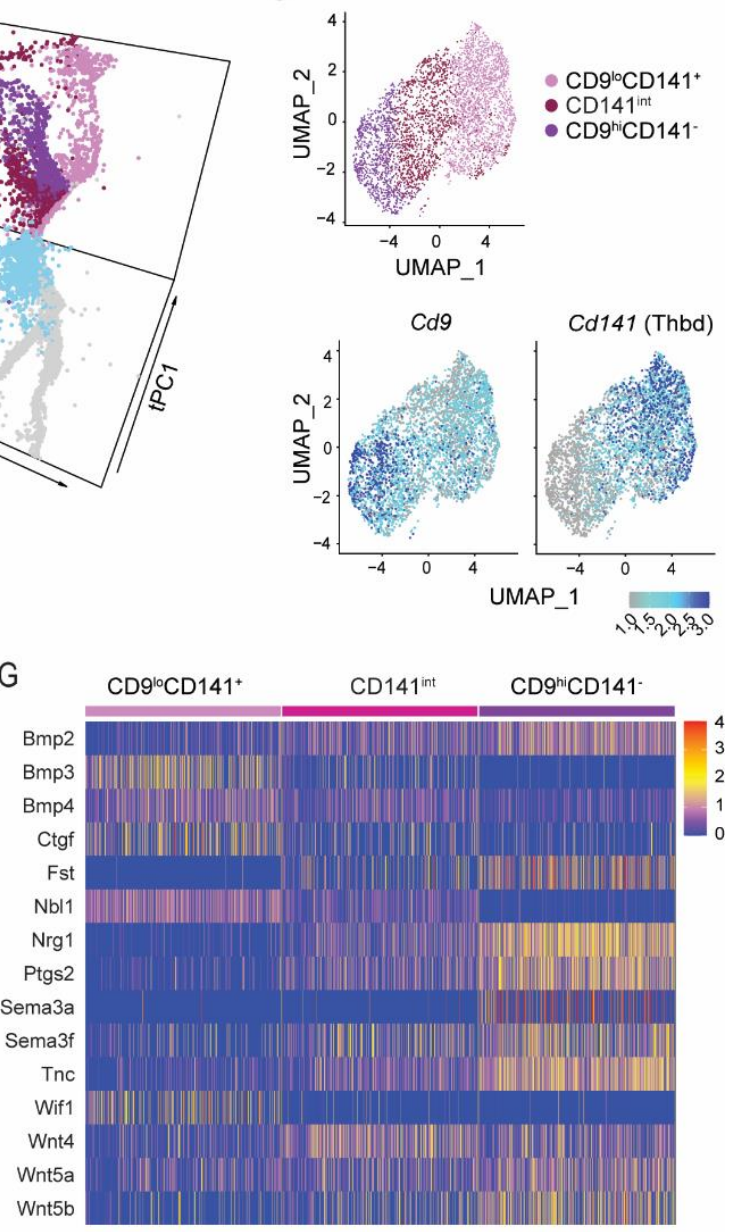

457 Figure 6. Subepithelial PDGFRa $\alpha^{\text {hi }}$ FB consist of three transcriptionally distinct clusters

458 originating from Fgfr2 ${ }^{+}$FB. (A) tSPACE projection of adult colonic MSC in tPC1 and 3

highlighting Fgfr2 ${ }^{+}$FB and PDGFR $\alpha^{\text {hi }}$ FB overlaid with RNA Velocity. (B) tSPACE 

clusters. (C) UMAP dimensionality reduction of re-clustered colonic PDGFR $\alpha^{\text {hi }}$ FB (top panel), with $C d 9$ (bottom left panel) and $C d 141$ (bottom right panel). (D) Representative flow cytometric analysis of CD9 and CD141 expression by colonic PDGFR $\alpha^{\text {hi }}$ FB. Representative

464 plots from 2 experiments with 3 mice/experiment. (E) Acta2 ( $\alpha$ SMA) gene expression

465 projected onto UMAP of colonic PDGFR $\alpha^{\text {hi }}$ FB. (F) Immunohistochemical staining of 466 colonic tissue for indicated antigens. R1',', and R2',', represent magnifications of R1 and R2

467 quadrants (yellow squares) on left image. Results are representative stains from 3

468 experiments with 3 mice/experiment. Arrows indicate $\alpha \mathrm{SMA}^{+} \mathrm{PDGFR} \alpha^{\text {hi }} \mathrm{FB}$. (G) Heatmap 469 showing scaled transcription levels (integrated data) of significantly $(\mathrm{p}<0.05)$ differentially 470 expressed epithelial support genes between the PDGFR $\alpha^{\text {hi }}$ FB clusters. See also

471 Supplementary Fig. 6.

472

\section{Discussion}

474 Recent studies have demonstrated considerable heterogeneity within the intestinal LP MSC 475 compartment ${ }^{5-7,11,22,30}$ and suggested non-redundant roles for MSC subsets in intestinal homeostasis $7,8,11,30,55$, inflammation ${ }^{5,23,56}$ and cancer ${ }^{22}$. As these studies have largely focused

477 on single regions of the intestine $5-8,11,22,23,30,55$, it has been unclear whether there are

478 regionally circumscribed differences in the composition of LP MSC subsets along the length 479 of the intestine. By performing scRNA-seq analysis of small intestinal and colonic LP MSC 480 from the same mice, we show here that both locations contain similar LP MSC subsets and 481 that their pattern of expression of epithelial support genes by LP MSC subsets is largely 482 conserved between these sites. Bulk RNA-seq analysis of sorted PDGFR $\alpha^{\text {hi }}$ subepithelial FB and interstitial $\mathrm{Fgfr}^{+} \mathrm{FB}$ confirmed that these subsets expressed distinct arrays of epithelial support genes irrespective of the tissue. However, both PDGFR $\alpha^{\text {hi }}$ and Fgfr2 $2^{+} \mathrm{FB}$ expressed 
higher levels of many epithelial support genes in the colon compared with their small intestinal counterparts, indicating a greater role for these FB in sustaining epithelial integrity in the colon. Consistent with this idea, WNT secretion by Gli1-expressing MSC is essential for homeostasis of the colonic epithelium ${ }^{6,15,55}$, but this is not the case in the small intestine where Paneth cells represent a major source of WNTs ${ }^{57}$.

Among the pathways significantly upregulated in colonic FB subsets compared with those in small intestine were TGF $\beta$ regulation of extracellular matrix expression, epidermal growth factor receptor (EGFR1) signaling, brain derived neurotrophic factor (BDNF) signaling pathway and thyroid stimulating hormone (TSH) regulation of gene expression. The relevance of these pathways in colonic versus small intestinal homeostasis remains to be determined. In contrast, few pathways were selectively upregulated in small intestinal FB subsets. Among these, several genes encoding chemokines, cytokines and cytokine receptors were significantly overexpressed by the Fgfr2 $2^{+} \mathrm{FB}$ population, suggesting that this subset may be involved in immune functions in the small intestine, but not in the colon. Interestingly, small intestinal FB also expressed higher levels of enzymes involved in vitamin A metabolism, consistent with previous findings that some small intestinal FB display aldehyde dehydrogenase activity and that there is increased retinoic acid receptor signaling in the small compared with the large intestine ${ }^{18,58}$. Collectively, these findings indicate that the local microenvironment plays a crucial role in regulating the transcriptional profile and specialization of intestinal FB in different regions of the intestine. The nature of the relevant factors and their importance in local homeostasis awaits further study.

Consistent with the idea that they may provide niche-specific support for local cells, the subsets of intestinal FB were located within distinct regions of the gut wall. As others have shown ${ }^{10,31,59}$, we found that PDGFR $\alpha^{\text {hi }}$ FB directly underlie the intestinal epithelium in 
510 both the small and large intestine. In contrast to an earlier report that small intestinal CD81 ${ }^{+}$

511 FB lie solely within the submucosa ${ }^{16}$, we found these cells within both the submucosa and

512 surrounding larger vessels deep in the mucosa, consistent with more recent studies ${ }^{7,11}$. In

513 addition, we demonstrate that $\mathrm{CD} 81^{+} \mathrm{FB}$ are found in similar locations in the small intestine

514 and colon. CD81 ${ }^{+} \mathrm{FB}$ play an essential role in maintaining the epithelial stem cell niche in the

515 small intestine, partly through their selective expression of the BMP antagonist, gremlin-1 ${ }^{7}$.

516 Consistent with this, our scRNA-seq analysis demonstrated that CD81 ${ }^{+} \mathrm{FB}$ were the major

517 source of Grem1 in the small intestine, although $\mathrm{CD} 81^{+} \mathrm{FB}, \mathrm{CD} 90^{+} \mathrm{FB}$ and $\mathrm{Fgfr} 2^{+} \mathrm{FB}$ all

518 expressed Grem 1 in the colon, indicating potential redundancy between these subsets in

519 supporting the colonic epithelial stem cell niche.

520 Less is known regarding the location of the intestinal PDGFR $\alpha^{\text {lo }}$ FB subsets that do not express CD81. Our immunohistochemical analysis demonstrated that $\mathrm{Fgfr} 2^{+} \mathrm{FB}$ were

522 located preferentially within the villus core towards the villus tip in the small intestine, while

523 those in the colon were located between crypts. These findings are consistent with work on

524 Fgfr2-mCherry reporter mice that suggested the Fgfr $2^{+}$cells represent interstitial $\mathrm{FB}^{22}$.

525 Using PPAR $\gamma$ expression as a surrogate marker, we could show that $\mathrm{CD}^{+} 0^{+}\left(\mathrm{PPAR} \gamma^{+}\right) \mathrm{FB}$ were located near the base of colonic crypts, but were unable to do this in the small intestine, as we failed to identify a specific marker for this population. However, small intestinal $\operatorname{Igfbp}^{+} \mathrm{FB}$ and colonic $\mathrm{CD} 90^{+} \mathrm{FB}$ resembled one another transcriptionally and Igfbp5 ${ }^{+} \mathrm{FB}$ were the only $\mathrm{CD} 34^{+} \mathrm{CD} 81^{-} \mathrm{FB}$ subset in small intestine. As the cells with this phenotype 530 were located primarily around small intestinal crypts, these results indicate that CD90 ${ }^{+} \mathrm{FB}$ and Igfbp5 $5^{+} \mathrm{FB}$ appear to represent a peri-cryptal population of FB in the colon and small intestine, respectively. Collectively, our findings confirm and extend previous work on the

533 localization of intestinal FB subsets, highlighting their distinct transcriptional profiles and 534 complex spatial organization within the mucosa. 

organs, including the serosal surface of the intestine ${ }^{60}$. Mesothelial cells undergo epithelialmesenchymal transition (EMT) and can give rise to both SMC and FB in response to injury in a number of tissues including the intestine ${ }^{26,28,61}$. During ontogeny, the intestinal mesothelium is a source of precursors for SMC in the intestinal muscle layers and vasculature

$540{ }^{27}$, as well as for uncharacterized FB in the outer serosa of the intestine ${ }^{28}$. In contrast, the origin(s) of the MSC subsets in the adult small intestine and colon LP has remained unclear.

542 Here we used intestinal transplantation and lineage-tracing approaches to demonstrate that all

543 adult small intestine and colon LP MSC subsets derive from Glil-expressing progenitors

544 present in the E12.5 intestine. At that time point, Glil expression was restricted to

545 mesothelial cells and an embryonic population of CD34-PDGFR $\alpha^{+} \mathrm{FB}$. scRNA-seq analysis

546 showed that both mesothelial cells and a minor cluster of cells within the CD34-PDGFR $\alpha^{+}$

547 FB population expressed markers previously associated with FB progenitors, while tSPACE

548 analysis suggested a direct trajectory connection between these two populations. These

549 results provide strong evidence that the mesothelium is a source of FB precursors during 550 early intestinal development and that these are capable of giving rise to all adult LP MSC 551 subsets. Interestingly, while also orignating from embryonic mesothelium, smooth muscle cells (SMC) in adult LP developed along an embryonic trajectory that was distinct from that of adult FB in tSPACE. Thus, SMC and FB in the steady state adult LP appear to represent

554 independent lineages that are specified during development. tSPACE analysis revealed direct connections between embryonic MSC clusters and adult $\mathrm{CD} 81^{+} \mathrm{FB}$, suggesting that adult $\mathrm{FB}$ subsets arise from $\mathrm{CD} 81^{+} \mathrm{FB}$, rather than from 557 distinct populations of intermediates that develop in the embryo. Consistent with this idea, $558 \mathrm{CD} 1^{+} \mathrm{FB}$ locate in the submucosa and in the adventitia surrounding larger vessels at the 559 base of the mucosa, an anatomical niche that contains MSC progenitors in other tissues ${ }^{41,62-}$ 
${ }^{67}$. Furthermore, lineage tracing of $\mathrm{Greml}^{+} \mathrm{FB}$ in the adult small intestine has identified progenitors that give rise to subepithelial FB along the entire crypt-villus axis, in a process that is relatively rapid during the perinatal period, but takes around a year to be completed in

563 adult intestine ${ }^{50}$. In support of this idea, we found that Greml expression is largely restricted 564 to $\mathrm{CD} 81^{+} \mathrm{FB}$ in the small intestine.

565 While early trajectory analysis suggested a bifurcation downstream from $\mathrm{CD} 81^{+} \mathrm{FB}^{5}$, 566 our tSPACE, pseudotime and Velocity analyses suggested that adult colonic CD81 ${ }^{+} \mathrm{FB}$

567 connected in a linear direction to adult $\mathrm{CD} 90^{+} \mathrm{FB}$, then to $\mathrm{Fgfr} 2^{+} \mathrm{FB}$ and finally to $\mathrm{PDGFR} \alpha^{\text {hi }}$

568 FB. Although we used different markers, our findings are consistent with those recently

569 published by Buechler et al who suggested that adventitial Pil6 ${ }^{+}$FB give rise first to Col15 $\alpha 1^{+} \mathrm{FB}$ and then to tissue specific FB clusters that in the intestine included $\mathrm{FblnI}^{+} \mathrm{FB}$

571 and subsequently $\mathrm{Bmp}^{+} \mathrm{FB}^{52}$. Overlaying these clusters on our tSPACE trajectories

572 demonstrated that the $\mathrm{Pill}^{+}, \mathrm{Coll} \mathrm{\alpha l}^{+}$and $\mathrm{FblnI}^{+} \mathrm{FB}$ most closely resembled the CD $81^{+}$,

$573 \mathrm{CD}^{+} 0^{+}$and $\mathrm{Fgfr} 2^{+} \mathrm{FB}$ we found in colon, while the $\mathrm{Bmp} 4^{+} \mathrm{FB}$ were similar to our colonic

574 PDGFR $\alpha^{\text {hi }}$ FB. The trajectory from $\mathrm{CD} 81^{+} \mathrm{FB}$ also correlates with the basal to apical

575 localization of the downstream subsets in the colonic LP, indicating that this process may

576 reflect factors present in distinct microenvironmental niches. Despite this strong evidence for

577 linear differentiation from a single precursor, it should be noted that FB show evidence of

578 slow turnover in the adult intestine ${ }^{5,30,50}$ and we cannot exclude the possibility that each FB

579 subset might self-renew in situ.

580 Recent scRNA-seq studies have suggested that colonic subepithelial PDGFR $\alpha^{\text {hi }}$ FB 581 are heterogeneous ${ }^{5,22}$ and here we found that PDGFR $\alpha^{\text {hi }}$ FB diverged into 3 clusters, which 582 we could define as $\mathrm{CD} 9{ }^{\mathrm{lo}} \mathrm{CD} 141^{+}, \mathrm{CD} 9{ }^{\mathrm{hi}} \mathrm{CD} 141^{-}$and $\mathrm{CD} 141^{\text {int }} \mathrm{FB}$. The $\mathrm{CD} 9{ }^{\mathrm{lo}} \mathrm{CD} 141^{+} \mathrm{FB}$ are 583 likely related to the PDGFR $\alpha^{\text {hi }}$ FB sub-cluster S2a defined by Kinchen et al, as they 584 expressed high levels of $\mathrm{Cxcl12}$, while $\mathrm{CD}{ }^{\text {hi }} \mathrm{CD} 141^{-} \mathrm{FB}$ expressed high levels of $\mathrm{Nrg} 1$ and 
585

586

587

588

589

590

591

592

593

594

595

596

597

598

599

600

601

602

603

604

605

606

607

608

609

so are likely related to the PDGFR $\alpha^{\text {hi }}$ FB subcluster S2b ${ }^{5}$. Although all three clusters expressed the telocyte marker Foxl1 ${ }^{10}, \mathrm{CD} 9{ }^{\mathrm{lo}} \mathrm{CD} 141^{+} \mathrm{FB}$ and to a lesser extent $\mathrm{CD} 141^{\text {int }} \mathrm{FB}$, expressed Acta2, coding for $\alpha$-smooth muscle actin ( $\alpha$ SMA), a marker often associated with myofibroblasts. $\alpha$ SMA ${ }^{+}$PDGFR $\alpha^{\text {hi }}$ FB were located directly underneath the epithelium approximately half way up colonic crypts, suggesting that CD $9{ }^{\text {lo }} \mathrm{CD} 141^{+}$and $\mathrm{CD} 9{ }^{\mathrm{hi}} \mathrm{CD} 141^{-}$ subepithelial FB localise within distinct regions along the crypt axis. Of the three PDGFR $\alpha^{\text {hi }}$ FB clusters, $\mathrm{CD} 9{ }^{\mathrm{lo}} \mathrm{CD} 141^{+} \mathrm{FB}$ expressed the highest levels of the WNT antagonists Wif1, Bmp3 and Bmp4. Therefore we speculate that the location of $\mathrm{CD} 9{ }^{\text {lo }} \mathrm{CD} 141^{+} \mathrm{FB}$ half way up colonic crypts allows them to promote the terminal differentiation of epithelial cells as they migrate up the crypt ${ }^{68}$. In contrast, $\mathrm{CD} 9{ }^{\text {hi }} \mathrm{CD} 141^{-} \mathrm{FB}$ expressed high levels of top of cryptassociated non-canonical Wnt4, Wnt5a and Wnt5b ${ }^{69,70}$ and Tenascin $C(T n c)^{71,72}$ and base of crypt-associated Ptgs 2 (the gene encoding COX-2) ${ }^{14,22}, \operatorname{Sema}_{3} a^{15}$. Thus, our findings indicate that each of the three PDGFR $\alpha^{\text {hi }}$ FB subsets may play distinct roles in colonic epithelial homeostasis.

In conclusion, our study provides a comprehensive mapping of intestinal MSC diversity, location and epithelial support function and highlights a central role for location along the intestinal length in regulating transcriptional profile and functional specialization. We also show that all adult MSC derive from Gli1-expressing embryonic mesothelial cells and we propose there is a linear developmental relationship between adult FB subsets that culminates in the development of a heterogeneous group of subepithelial PDGFR $\alpha^{\text {hi }}$ FB. Together our findings provide key insights into MSC diversity, development, function and interrelationships with relevance to intestinal development and homeostasis.

\section{Methods}

Mice and ethical statements 
612 EYFP mice (obtained by crossing R26R.EYFP with the relevant Cre mice) were bred and

613 maintained at the Bio-Facility animal house (Technical University of Denmark). C57BL/6Nrj

614 mice were purchased from Janvier Labs (Le Genest-Saint-Isle, France). Ackr $4^{\mathrm{tmlCcbl1}}$ mice

615 (Ackr4.EGFP) ${ }^{74}$ were bred and maintained in the Central Research Facility, Glasgow

616 University. Adult mice were used between 5.5 and 12w of age. Mice of both genders were

617 used in all experiments and littermates were used as controls. All experiments were approved

618 by the Danish Animal Experiments Inspectorate, or with ethical approval under a Project

619 Licence from the the UK Home Office.

620

621 Kidney grafting

622 EYFP male mice were mated overnight with C57BL/6Nrj females and the following morning 623 was defined as gestational day 0.5 (E0.5). Pregnant dams were sacrificed at E12.5 and small 624 and large intestine were dissected from embryos under a stereo microscope (VWR). Adult 625 WT mice were anaesthetized by i.p injection of Ketaminol Vet. (100mg/kg, MSD animal 626 health) and Rompun Vet. (10mg/kg, Bayer) and were injected subcutaneously with Bupaq $627(0.1 \mathrm{mg} / \mathrm{kg}$, Richter Pharma). Washed embryonic intestine was transplanted under the kidney 628 capsule of anesthetized recipients as described previously ${ }^{37}$. Recipients were sacrificed at the 629 time points indicated and grafts were dissected and cut into pieces prior to cell isolation as 630 described below.

631

632 In vivo lineage tracing

633 Glil-CreER ${ }^{\mathrm{T} 2}$ male mice were mated overnight with $R 26 R$.EYFP females and the following 634 morning was defined as gestational day E0.5. At E11.5, pregnant dams were injected i.p. with 
637 Scientific). Small and large intestine were isolated from embryos or weaned offspring at the 638 time points indicated.

639

\section{Cell isolation}

641 Intestinal cell suspensions were generated as described previously ${ }^{75}$ with minor changes.

642 Briefly, washed intestinal tissue was opened longitudinally and Peyer's patches removed. For

643 scRNA-seq and bulk RNA-seq experiments on adult intestine, muscularis externa was

644 stripped away using tweezers. Tissues were cut into $0.5-1 \mathrm{~cm}$ pieces and epithelial cells

645 removed by 3 consecutive rounds of incubation in HBSS supplemented with HEPES

$646(15 \mathrm{mM})$, sodium pyruvate $(1 \mathrm{mM})$, penicillin/streptomycin $(100 \mathrm{U} / \mathrm{mL})$, gentamycin $(0.05$

$647 \mathrm{mg} / \mathrm{mL})$, EDTA (2mM) (all Invitrogen) and FCS (2.5\%) (Sigma), for $15 \mathrm{~min}$ at $37^{\circ} \mathrm{C}$ with

648 constant shaking at $350 \mathrm{rpm}$. After each incubation, samples were shaken for $10 \mathrm{sec}$ and

649 medium containing epithelial cells and debris was discarded. For colonic tissues, DL-

650 dithiothreitol (5mM) (Sigma) was added at the first incubation step. Remaining tissue pieces

651 were digested with collagenase P $(0.6 \mathrm{U} / \mathrm{mL}$, Sigma $)$ or with Liberase TM $(0.325 \mathrm{U} / \mathrm{mL}$,

652 Roche) and DNAse I (31 $\mathrm{g} / \mathrm{mL}$, Roche) in R10 medium (RPMI 1640, sodium pyruvate

653 (1mM), HEPES $(10 \mathrm{mM})$, penicillin/streptomycin $(100 \mathrm{U} / \mathrm{mL})$, gentamycin $(0.05 \mathrm{mg} / \mathrm{mL})$,

654 and $10 \%$ FCS) for up to $30 \mathrm{~min}$ at $37^{\circ} \mathrm{C}$ with constant shaking at $550 \mathrm{rpm}$ (small intestine) or 655 with a magnetic stirrer and at $280 \mathrm{rpm}$ (large intestine). For bulk RNA-seq cells were treated 656 with ACK lysing buffer (Gibco) to lyse red blood cells prior to sorting. For isolation of cells

657 from embryonic intestine, tissues were digested directly for $30 \mathrm{~min}$ at $37^{\circ} \mathrm{C}$ in Eppendorf

658 tubes with constant shaking at $900 \mathrm{rpm}$. The resulting cell suspensions were filtered through a 
$70 \mu \mathrm{m}$ filter and washed in MACS buffer (PBS with 3\% FCS and 2 mM EDTA) twice prior

660 to subsequent analyses.

661

\section{Flow cytometry and cell sorting}

663 Cell suspensions were stained with fluorochrome labelled primary antibodies (see Table 1) in 664 Brilliant stain buffer (BD Biosciences) for 30 min on ice. Flow cytometry was performed on 665 an LSR Fortessa II (BD Biosciences), FACSAria Fusion (BD Biosciences), or FACSMelody

666 (BD Biosciences) and analysed with FlowJo software (TreeStar). Dead cells were identified by staining with either 7-AAD (eBioscience) or Zombie UV fixable viability dye (BD Biosciences) and cell doublets were excluded on the basis of FSC-A/FSC-H. For intracellular staining, cells were stained for surface antigens, fixed with FoxP3 Staining Buffer set

670 (eBioscience) and stained for $\alpha$ SMA in FoxP3 Permeabilization buffer (eBioscience). After

671 washing, cells were stained with antibodies to surface antigens not compatible with fixation according to the manufacturer's instructions.

673

674 Immunohistochemistry

675 Tissues were fixed in paraformaldehyde (4\%, PFA) and sectioned (70 $\mu \mathrm{m})$ using a Vibratome 676 (Leica VT12000S). Sections were incubated in PBS containing bovine serum albumin (BSA) $677(1 \%)$ and Triton-X100 (0.3\%) for 1 hour at room temperature (RT) to block non-specific 678 staining and incubated with fluorochrome conjugated or unconjugated primary antibodies 679 (see Table 1) overnight at $4^{\circ} \mathrm{C}$. After washing with PBS containing Triton-X100 (0.3\%), 680 tissues were incubated with secondary antibodies (see Table 1) at RT for 2-4 hours. For detection of CD81, staining with biotinylated anti-CD81 was enhanced using the Biotinyl Tyramide kit (Perkin Elmer) according to the manufacturer's instructions after blocking of endogenous biotin using Streptavidin/Biotin Blocking kit (Invitrogen). 
684 Endogenous peroxidase was inactivated by incubating tissues with $3 \% \mathrm{H}_{2} \mathrm{O}_{2}$ for 30 min at RT

685

686

687

688

689

690

691

692

693

694

695

696

697

698

699

700

701

702

703

704

705

706

707

708 before incubation with streptavidin-horse radish peroxidase (HRP). Sections were analysed under 40x magnification using a Zeiss LSM 710 confocal microscope and the images were processed using Zeiss Zen and Imaris software.

For histological analysis of kidney grafts, tissue pieces were fixed in $4 \%$ paraformaldehyde for $8 \mathrm{~h}$ and paraffin-embedded sections were stained with hematoxylin and eosin.

\section{Library Preparation and sequencing}

Single cell RNA-seq. Sorted cells were washed in cold PBS containing bovine serum albumin $(0.04 \%)$, counted and diluted to the desired concentration following 10X Genomics guidelines (10x Genomics, CG000053_CellPrepGuide_RevC). ScRNA-seq libraries were prepared according to the manufacturer's instructions using Chromium Single Cell 3' Library

\& Gel Bead Kit v3 (10x Genomics, PN-1000092) or 5' kit Chromium Single Cell 5' Library \& Gel Bead Kit (10x Genomics, PN-1000006) and Chromium Chip B Single Cell Kit (PN1000074) with the Chromium Controller \& Next GEM Accessory Kit (10x Genomics, PN120223). In brief, single cells, reverse transcription reagents, Gel Beads containing barcoded oligonucleotides, and oil were combined on a microfluidic chip to form Gel Beads in Emulsion (GEMs). Individual cells were lysed inside the GEMs and the released poly-A transcripts were barcoded with an Illumina R1 sequence, a 10X barcode and a Unique Molecular Identifier (UMI) during reverse transcription (RT). After RT, GEMs were broken, barcoded cDNA was purified using Dynabeads MyOne silane (10x Genomics, PN-2000048) and amplified by Polymerase Chain Reaction (PCR). Amplified cDNA were cleaned up with SPRIselect Reagent kit (Beckman Coulter, B23318). Indexed sequencing libraries were constructed by enzymatic fragmentation, end-repair and A-tailing, before a second and final PCR amplification using the Chromium i7 Sample Index (10x Genomics, PN-220103), 
709

710

711

712

713

714

715

716

717

718

719

720

721

722

723

724

725

726

727

728

729

730

731

732

733

introducing an Illumina R2 sequence, a unique sample index (allowing multiplex sequencing) and P5/P7 Illumina sequencing adaptors to each library. Library quality control and quantification were performed using a KAPA Library Quantification Kit for Illumina Platforms (Kapa Biosystems, KK4873) and the 2100 Bioanalyzer equipped with a High Sensitivity DNA kit (Agilent, 5067-4626). Muliplexed libraries were pooled and sequenced either by NextSeq 500/550 High Output v2.5 kit (150 cycles) at the Center of Excellence for Fluorescent Bioanalytics (KFB, University of Regensburg, Germany) or by Novaseq 6000 S1 or S2 (200 cycles) at the SNP\&SEQ Technology Platform (Uppsala, Sweden) with the following sequencing run parameters: Read1 - 28 cyles; i7 index - 8 cycles; Read2 - 126 cycles at a depth of at least 100M reads/sample.

Bulk RNA-seq. MSC subsets were sorted into RLT buffer and total RNA was isolated using the RNeasy Micro kit (Qiagen). Following the manufacturer's protocol, extraction was performed with an on-column DNAse digestion step after the first washing step. The RNA quality and quantity were measured using the 2100 BioAnalyzer equipped with RNA6000 Pico chip (Agilent Technologies). Using Ovation RNA-Seq System V2 kit (Nugen), RNA was subjected to whole transcriptome amplification and the MiniElute Reaction Cleanup kit (Qiagen) was used to purify the amplified cDNA samples. The quantity and quality of the cDNA samples were measured using the 2100 BioAnalyzer equipped with DNA1000 chip (Agilent technologies) and the Nanodrop (ThermoFisher Scientific). Following the manufacturer's instructions, libraries were constructed with the Ovation Ultralow system V2 kit (Nugen). A Bioruptor Pico (Diagenode) was used to fragment amplified cDNA (100 ng) by sonication, and sheared cDNA end-repaired to generate blunt ends and ligated to Illumina adaptors with indexing tags followed by AMPure XP bead purification. A 2100 Bioanalyzer equipped with DNA1000 chip (Agilent technologies) was used to evaluate library size 
distribution, and this was quantified using KAPA library Quantification Kit Illumina platforms (Kapa Biosystems). Libraries were diluted before being pooled at equimolar concentration (10 $\mathrm{nM}$ final) and subsequently sequenced on the Hiseq2500 platform (Illumina) using 50bp single reads (Center for Genomic Regulation, Spain) with a read depth of $15-20 \mathrm{M}$ reads per sample.

\section{Computational analysis}

Single cell RNA-seq. Alignment of scRNA-seq data to mouse reference genome, mm10, was performed with CellRanger (version 3.0.2 \& 3.1.0) ${ }^{76,77}$. The data was imported into $\mathrm{R}$ (version 4.0.1) ${ }^{78}$ and processed to remove debris and doublets in individual samples by looking at gene, read counts and mitochondrial gene expression. Variable genes were calculated with Seurats FindVariableFeatures function and selection method set to "vst" (Seurat version 3.1.5) ${ }^{79}$. The respective samples and all their overlapping genes were then integrated with anchor integration for Seurat. Cell cycle effects were regressed out with linear regression using a combination of the build-in function in Seurat and scoring gene sets from ccremover per cell ${ }^{80}$ during scaling of the gene expression. The datasets were dimensionality reduced first with PCA and then UMAP and clustered with Louvain clustering all using Seurat. After initial clustering, contaminating cells were removed and an additional round of clustering and dimensionality reduction with UMAP was run on the cells of interest. DEGs were identified using Seurat FindAllMarkers function with the default test setting (nonparameteric Wilcoxon Rank Sum test). Expression of gene modules in the form of published signature gene sets were calculated with AddModuleScore (Seurat) taking the top DEG from telocytes (10 genes), Lo-1 FB (10 genes) and Lo-2 FB (7 genes) reported by McCarthy et $a l^{7}$, the top 10 DEG from FB1-5, MC and SMCs reported by Hong et al ${ }^{11}$, and the top 20 DEG from $\mathrm{Pil6}^{+}, \mathrm{Coll}_{\mathrm{a}} \mathrm{I}^{+}, \mathrm{FblnI}^{+}$and $\mathrm{Bmp}^{+} \mathrm{FB}$ reported by Buechler et al ${ }^{52}$. Pearson 
correlations between datasets were calculated based on average expressions per cluster of overlapping variable genes and plotted with heatmap.2 (version 3.0.3) ${ }^{81}$. Heatmaps were constructed with a modified version of Seurats DoHeatmap to allow for multiple grouping variables. Data plotted in expression heatmaps was scaled based on the anchor integrated data. Data imputation was performed per dataset across samples on raw count data with magicBatch ${ }^{44}$ where the affinity matrix used was Seurat's batch-corrected PCA coordinates. Trajectories and trajectory spaces were determined with tSPACE ${ }^{43}$ on the top 2000 imputed variable genes for adult trajectories and top 1000 imputed variable genes for the integrated E12.5 and adult trajectory. GO analysis was performed using GO Biological Process $2018^{53}$

768 from Enrichr computational biosystems ${ }^{82-84}$.

769 Bulk RNA-seq. Raw RNA sequencing data from the 30 samples were pre-processed with TrimGalore (version 0.4.0) and FastQC (version 0.11.2). Pseudo-alignment of reads was performed with Kallisto (version 0.42 .5 ) to obtain RNA expression information. To assess correlations between bulk-seq samples and SC clusters Pearson correlations based on SC variable genes were calculated between the bulk-seq samples and the pseudo-bulk of the SC

774 clusters for the individual tissues and visualized with heatmap.2 (part of gplot package).

775 For all DESeq2 $\left(1.26 .0^{85}\right)$ analysis, transcripts identified in less than 3 replicates and at levels below 6 reads were filtered out prior to further analysis. Heatmaps of bulk-seq data expression was created in $\mathrm{R}$ with the ComplexHeatmap package (version 2.7.11) and volcano

778 plots with ggplot2 (version 3.3.1). For the comparison between tissues, DEGs were only 779 classified as significant if they had a $\left|\log _{2} \mathrm{FC}\right|>1.5$ and adjusted p-value $<0.05$. GO analysis was performed using BioPlanet $2019^{33}$ from Enrichr computational biosystems ${ }^{82-84}$. 
783 Statistical significance was determined with a 2-way ANOVA with Benjamini, Krieger and

784 Yekutieli multiple comparisons and performed in Prism software (GraphPad). ${ }^{*} \mathrm{p}<0.05,{ }^{* *} \mathrm{p}$ $785<0.01, * * * \mathrm{p}<0.001$

786

787 Lead Contact

788 Further information and requests for resources and reagents should be directed to and will be 789 fulfilled by the Lead Contact, Dr WW Agace (wiag@dtu.dk).

790

\section{Data Availability}

792 Single-cell RNA-seq and bulk RNA-seq data has been deposited at NCBI GEO and can be 793 found under the accession number GSE182176 containing all datasets (Small and large 794 intestine scRNA-seq data (adult)), E12.5 large intestine scRNA-seq dataset, kidney graft 795 large intestine scRNA-seq dataset, small and large intestine MSC bulk RNA-seq dataset). Microscopy data reported in this paper will be shared by the lead contact upon request. This paper does not report original code. Any additional information required to re-analyze the 798 data reported in this paper is available from the lead contact upon request.

799

800

\section{Supplementary Information}

801 The manuscript contains 6 supplemental Figures and 3 supplemental Tables.

802

803 Supplemental Table 1: List of genes that are differentially expressed between small 804 intestinal and colonic PDGFR $\alpha^{\text {hi }}$ FB ranked in order of significance. Included genes have a $805|\log 2 \mathrm{FC}|>1.5$ and adjusted $\mathrm{p}$-value $<0.05$. 
807 Supplementary Table 2. List of genes that are differentially expressed between small 808 intestinal and colonic Fgfr $2^{+} \mathrm{FB}$ ranked in order of significance. Included genes have a $809|\log 2 \mathrm{FC}|>1.5$ and adjusted $\mathrm{p}$-value $<0.05$.

810

811 Supplementary Table 3. List of common genes that are differentially expressed between 812 both small intestinal and colonic PDGFR $\alpha^{\text {hi }} \mathrm{FB}$ and small intestinal and colonic Fgfr2 ${ }^{+}$FB.

813 Included genes have a $|\log 2 \mathrm{FC}|>1.5$ and adjusted $\mathrm{p}$-value $<0.05$.

814

\section{References}

816 1. Mowat, A. M. \& Agace, W. W. Regional specialization within the intestinal immune 817 system. Nat. Rev. Immunol. 14, 667-685 (2014).

818 2. Agace, W. W. \& McCoy, K. D. Regionalized Development and Maintenance of the 819 Intestinal Adaptive Immune Landscape. Immunity 46, 532-548 (2017).

820 3. Parikh, K. et al. Colonic epithelial cell diversity in health and inflammatory bowel disease. Nature 1 (2019) doi:10.1038/s41586-019-0992-y.

822 4. Barker, N. Adult intestinal stem cells: Critical drivers of epithelial homeostasis and regeneration. Nat. Rev. Mol. Cell Biol. 15, 19-33 (2014).

824 5. Kinchen, J. et al. Structural Remodeling of the Human Colonic Mesenchyme in 825 Inflammatory Bowel Disease. Cell 175, 372-386.e17 (2018).

826 6. Degirmenci, B., Valenta, T., Dimitrieva, S., Hausmann, G. \& Basler, K. GLI1expressing mesenchymal cells form the essential Wnt-secreting niche for colon stem cells. Nature 558, 449-453 (2018).

829 7. McCarthy, N. et al. Distinct Mesenchymal Cell Populations Generate the Essential 830 Intestinal BMP Signaling Gradient. Cell Stem Cell 26, 391-402.e5 (2020).

8. Wu, N. et al. MAP3K2-regulated intestinal stromal cells define a distinct stem cell 
niche. Nature (2021) doi:10.1038/s41586-021-03283-y.

9. Brügger, M. D., Valenta, T., Fazilaty, H., Hausmann, G. \& Basler, K. Distinct populations of crypt-associated fibroblasts act as signaling hubs to control colon homeostasis. PLoS Biol. 18, 1-20 (2020).

10. Shoshkes-Carmel, M. et al. Subepithelial telocytes are an important source of Wnts that supports intestinal crypts. Nature 557, 242-246 (2018).

11. Hong, S. P. et al. Distinct fibroblast subsets regulate lacteal integrity through YAP/TAZ-induced VEGF-C in intestinal villi. Nat. Commun. 11, 4102 (2020).

12. Roulis, M. \& Flavell, R. A. Fibroblasts and myofibroblasts of the intestinal lamina propria in physiology and disease. Differentiation 92, 116-131 (2016).

13. Furuya, S. \& Furuya, K. Subepithelial Fibroblasts in Intestinal Villi: Roles in Intercellular Communication. Int. Rev. Cytol. 264, 165-223 (2007).

14. Stzepourginski, I. et al. CD34 + mesenchymal cells are a major component of the intestinal stem cells niche at homeostasis and after injury. Proc. Natl. Acad. Sci. 114, E506-E513 (2017).

15. Karpus, O. N. et al. Colonic CD90+ Crypt Fibroblasts Secrete Semaphorins to Support Epithelial Growth. Cell Rep. 26, 3698-3708.e5 (2019).

16. Thomson, C. A. et al. Expression of the Atypical Chemokine Receptor ACKR4 Identifies a Novel Population of Intestinal Submucosal Fibroblasts That Preferentially Expresses Endothelial Cell Regulators. J. Immunol. 201, 215-229 (2018).

17. Fawkner-Corbett, D. et al. Spatiotemporal analysis of human intestinal development at single-cell resolution. Cell 1-17 (2021) doi:10.1016/j.cell.2020.12.016.

18. Vicente-Suarez, I. et al. Unique lamina propria stromal cells imprint the functional phenotype of mucosal dendritic cells. Mucosal Immunol. 8, 141-151 (2015).

19. Fagarasan, S., Kinoshita, K., Muramatsu, M., Ikuta, K. \& Honjo, T. In situ class 
switching and differentiation to IgA-producing cells in the gut lamina propria. Nature 413, 639-643 (2001).

20. Beswick, E. J. et al. TLR4 Activation Enhances the PD-L1-Mediated Tolerogenic Capacity of Colonic CD90+ Stromal Cells. J. Immunol. 193, 2218-2229 (2014).

21. Powell, D. W., Pinchuk, I. V., Saada, J. I., Chen, X. \& Mifflin, R. C. Mesenchymal Cells of the Intestinal Lamina Propria. Annu. Rev. Physiol. 73, 213-237 (2011).

22. Roulis, M. et al. Paracrine orchestration of intestinal tumorigenesis by a mesenchymal niche. Nature 580, 524-529 (2020).

23. Smillie, C. S. et al. Intra- and Inter-cellular Rewiring of the Human Colon during Ulcerative Colitis. Cell 178, 714-730.e22 (2019).

24. Aoki, R. et al. Foxl1-Expressing Mesenchymal Cells Constitute the Intestinal Stem Cell Niche. Cell. Mol. Gastroenterol. Hepatol. 2, 175-188 (2016).

25. Holloway, E. M. et al. Mapping Development of the Human Intestinal Niche at SingleCell Resolution. Cell Stem Cell 28, 568-580.e4 (2021).

26. Koopmans, T. \& Rinkevich, Y. Mesothelial to mesenchyme transition as a major developmental and pathological player in trunk organs and their cavities. Commun. Biol. 1, 170 (2018).

27. Wilm, B., Ipenberg, A., Hastie, N. D., Burch, J. B. E. \& Bader, D. M. The serosal mesothelium is a major source of smooth muscle cells of the gut vasculature. Development 132, 5317-5328 (2005).

28. Rinkevich, Y. et al. Identification and prospective isolation of a mesothelial precursor lineage giving rise to smooth muscle cells and fibroblasts for mammalian internal organs, and their vasculature. Nat. Cell Biol. 14, 1251-1260 (2012).

29. Taylor, R. T. et al. Lymphotoxin-Independent Expression of TNF-Related ActivationInduced Cytokine by Stromal Cells in Cryptopatches, Isolated Lymphoid Follicles, and 
882

883

884

885

886

887

888

889

890

891

892

893

894

895

896

897

898

899

900

901

902

903

904

905

906

Peyer's Patches. J. Immunol. 178, 5659-5667 (2007).

30. Bahar Halpern, K. et al. Lgr5+ telocytes are a signaling source at the intestinal villus tip. Nat. Commun. 11, 1936 (2020).

31. Kurahashi, M. et al. A novel population of subepithelial platelet-derived growth factor receptor $\alpha$-positive cells in the mouse and human colon. Am. J. Physiol. Gastrointest. Liver Physiol. 304, G823-34 (2013).

32. Yuasa, Y. Control of gut differentiation and intestinal-type gastric carcinogenesis. Nat. Rev. Cancer 3, 592-600 (2003).

33. Huang, R. et al. The NCATS BioPlanet - An integrated platform for exploring the universe of cellular signaling pathways for toxicology, systems biology, and chemical genomics. Front. Pharmacol. 10, 1-13 (2019).

34. Meyerholz, D. K., Lambertz, A. M. \& McCray, P. B. Dipeptidyl Peptidase 4 Distribution in the Human Respiratory Tract Implications for the Middle East Respiratory Syndrome. Am. J. Pathol. 186, 78-86 (2016).

35. Yanai, H. et al. Intestinal stem cells contribute to the maturation of the neonatal small intestine and colon independently of digestive activity. Sci. Rep. 7, 2-5 (2017).

36. Mosley, L. \& Klein, J. R. Peripheral engraftment of fetal intestine into athymic mice sponsors T cell development: direct evidence for thymopoitic function of murine small intestine. J Exp Med 176, 1365-1373 (1992).

37. Ferguson, A., Parrott, M. V \& Connor, O. Growth and development of 'antigen-free' grafts of foetal mouse intestine. J. Pathol. 106, (1972).

38. Lee, M. Y. et al. Transcriptome of interstitial cells of Cajal reveals unique and selective gene signatures. PLoS One 12, (2017).

39. Namvar, S. et al. Functional molecules in mesothelial-to-mesenchymal transition revealed by transcriptome analyses. J. Pathol. 245, 491-501 (2018). 
40. Kanamori-Katayama, M. et al. LRRN4 and UPK3B are markers of primary mesothelial cells. PLoS One 6, 2-9 (2011).

41. Kramann, R. et al. Perivascular Gli1+ progenitors are key contributors to injuryinduced organ fibrosis. Cell Stem Cell 16, 51-66 (2015).

42. Srinivas, S. et al. Cre reporter strains produced by targeted insertion of EYFP and ECFP into the ROSA26 locus. BMC Dev Biol 1, 4 (2001).

43. Dermadi, D. et al. Exploration of Cell Development Pathways through HighDimensional Single Cell Analysis in Trajectory Space. iScience 23, 100842 (2020).

44. Brulois, K. magicBatch: R wrapper for the original python implementation of the Marcov Affinity-based Graph Imputation of Cells (MAGIC) algorithm. R package version 0.1.0. github (2020).

45. Xiang, M. et al. A Single-Cell Transcriptional Roadmap of the Mouse and Human Lymph Node Lymphatic Vasculature. Front. Cardiovasc. Med. 7, (2020).

46. Vallecillo-García, P. et al. Odd skipped-related 1 identifies a population of embryonic fibro-adipogenic progenitors regulating myogenesis during limb development. Nat. Commun. 8, (2017).

47. Dulauroy, S., Di Carlo, S. E., Langa, F., Eberl, G. \& Peduto, L. Lineage tracing and genetic ablation of ADAM12 + perivascular cells identify a major source of profibrotic cells during acute tissue injury. Nat. Med. 18, 1262-1270 (2012).

48. Castagnaro, L. et al. Nkx2-5+islet1+ mesenchymal precursors generate distinct spleen stromal cell subsets and participate in restoring stromal network integrity. Immunity 38, 782-791 (2013).

49. Bae, S. et al. Combined omics analysis identifies transmembrane 4 L6 family member 1 as a surface protein marker specific to human mesenchymal stem cells. Stem Cells Dev. 20, 197-203 (2011). 
932

933

934

935

936

937

938

939

940

941

942

943

944

945

946

947

948

949

950

951

952

953

954

955

956

50. Worthley, D. L. et al. Gremlin 1 identifies a skeletal stem cell with bone, cartilage, and reticular stromal potential. Cell 160, 269-284 (2015).

51. Driskell, R. R. et al. Distinct fibroblast lineages determine dermal architecture in skin development and repair. Nature 504, 277-281 (2013).

52. Buechler, M. B. et al. Cross-tissue organization of the fibroblast lineage. Nature (2021) doi:10.1038/s41586-021-03549-5.

53. Ashburner, M. et al. Gene Ontology: tool for the unification of biology. Nat. Genet. 25, 25-29 (2000).

54. La Manno, G. et al. RNA velocity of single cells. Nature 560, 494-498 (2018).

55. David, M. B., Valenta, T., Fazilaty, H., Hausmann, G. \& Basler, K. Distinct populations of crypt-associated fibroblasts act as signaling hubs to control colon homeostasis. PLoS Biol. 18, 1-20 (2020).

56. West, N. R. et al. Oncostatin M drives intestinal inflammation and predicts response to tumor necrosis factor-neutralizing therapy in patients with inflammatory bowel disease. Nat. Med. 23, 579-589 (2017).

57. Sato, T. et al. Paneth cells constitute the niche for Lgr5 stem cells in intestinal crypts. Nature 469, 415-418 (2011).

58. Jaensson-Gyllenbäck, E. et al. Bile retinoids imprint intestinal CD103+ dendritic cells with the ability to generate gut-tropic T cells. Mucosal Immunol. 4, 438-47 (2011).

59. Eyden, B., Curry, A. \& Wang, G. Stromal cells in the human gut show ultrastructural features of fibroblasts and smooth muscle cells but not myofibroblasts. J. Cell. Mol. Med. 15, 1483-1491 (2011).

60. Winters, N. I. \& Bader, D. M. Development of the serosal mesothelium. J. Dev. Biol. 1, 64-81 (2013).

61. Miyoshi, H., Ajima, R., Luo, C. T., Yamaguchi, T. P. \& Stappenbeck, T. S. Wnt5a 
Potentiates TGF- $\beta$ Signaling to Promote Colonic Crypt Regeneration After Tissue Injury. Science (80-. ). 338, 108-113 (2012).

62. Benias, P. C. et al. Structure and distribution of an unrecognized interstitium in human tissues. Sci. Rep. 8, 1-8 (2018).

63. Sitnik, K. M. et al. Context-Dependent Development of Lymphoid Stroma from Adult CD34+ Adventitial Progenitors. Cell Rep. 14, 2375-2388 (2016).

64. Kramann, R. et al. Adventitial MSC-like Cells Are Progenitors of Vascular Smooth Muscle Cells and Drive Vascular Calcification in Chronic Kidney Disease. Cell Stem Cell 19, 628-642 (2016).

65. Díaz-Flores, L. et al. Human resident CD34+ stromal cells/telocytes have progenitor capacity and are a source of $\alpha \mathrm{SMA}+$ cells during repair. Histol. Histopathol. 30, 615$627(2015)$.

66. Sidney, L. E., Branch, M. J., Dunphy, S. E., Dua, H. S. \& Hopkinson, A. Concise Review: Evidence for CD34 as a Common Marker for Diverse Progenitors. Stem Cells 32, 1380-1389 (2014).

67. Merrick, D. et al. Identification of a mesenchymal progenitor cell hierarchy in adipose tissue. Science (80-. ). 364, eaav2501 (2019).

68. Qi, Z. et al. BMP restricts stemness of intestinal Lgr5+ stem cells by directly suppressing their signature genes. Nat. Commun. 8, 13824 (2017).

69. Kosinski, C. et al. Gene expression patterns of human colon tops and basal crypts and BMP antagonists as intestinal stem cell niche factors. Proc. Natl. Acad. Sci. 104, $15418-15423$ (2007).

70. Gregorieff, A. et al. Expression pattern of Wnt signaling components in the adult intestine. Gastroenterology 129, 626-638 (2005).

71. Probstmeier, R., Martini, R. \& Schachner, M. Expression of J1/tenascin in the crypt- 
villus unit of adult mouse small intestine: Implications for its role in epithelial cell shedding. Development 109, 313-321 (1990).

72. Bernier-Latmani, J. et al. DLL4 promotes continuous adult intestinal lacteal regeneration and dietary fat transport. J. Clin. Invest. 125, 4572-4586 (2015).

73. Garcia, A. D. R., Petrova, R., Eng, L. \& Joyner, A. L. Sonic Hedgehog regulates discrete populations of astrocytes in the adult mouse forebrain. J. Neurosci. 30, 13597-13608 (2010).

74. Heinzel, K., Benz, C. \& Bleul, C. C. A silent chemokine receptor regulates steadystate leukocyte homing in vivo. Proc. Natl. Acad. Sci. U. S. A. 104, 8421-8426 (2007).

75. Schulz, O. et al. Intestinal CD103+, but not CX3CR1+, antigen sampling cells migrate in lymph and serve classical dendritic cell functions. J. Exp. Med. 206, 3101-3114 (2009).

76. Dobin, A. et al. STAR: Ultrafast universal RNA-seq aligner. Bioinformatics 29, 15-21 (2013).

77. Zheng, G. X. Y. et al. Massively parallel digital transcriptional profiling of single cells. Nat. Commun. 8, (2017).

78. R Core Team. R: A Language and Environment for Statistical Computing. $R$ Foundation for Statistical Computing (2020).

79. Stuart, T. \& Satija, R. Integrative single-cell analysis. Nat. Rev. Genet. 1 (2019) doi:10.1038/s41576-019-0093-7.

80. Li, Jun; Barron, M. ccRemover: Removes the Cell-Cycle Effect from Single-Cell RNA-Sequencing Data. CRAN (2017).

81. Gregory R. Warnes, Ben Bolker, Lodewijk Bonebakker, Robert Gentleman, Wolfgang Huber, Andy Liaw, Thomas Lumley, Martin Maechler, Arni Magnusson, Steffen Moeller, M. S. \& Venables, B. gplots: Various R Programming Tools for Plotting 
Data. CRAN (2020).

1008

1009

1010

1011

1012

1013

1014

1015

1016

1017

1018

1019

1020

1021

1022

1023

1024

1025

1026

1027

1028

1029

1030

1031

82. Kuleshov, M. V. et al. Enrichr: a comprehensive gene set enrichment analysis web server 2016 update. Nucleic Acids Res. 44, W90-W97 (2016).

83. Xie, Z. et al. Gene Set Knowledge Discovery with Enrichr. Curr. Protoc. 1, (2021).

84. Chen, E. Y. et al. Enrichr: interactive and collaborative HTML5 gene list enrichment analysis tool Edward. BMC Bioinformatics 128, 617-619 (2013).

85. Love, M. I., Huber, W. \& Anders, S. Moderated estimation of fold change and dispersion for RNA-seq data with DESeq2. Genome Biol. 15, 1-21 (2014).

\section{Acknowledgements}

We thank Dr. J. Vandamme (DTU, Denmark) for performing scRNA-seq and library preparation, Dr. A.L Joyner (Memorial Sloan-Kettering Cancer Center) for providing Gli1EGFP mice, Dr. S. Milling (University of Glasgow University, UK) for providing laboratory space and materials for experiments involving Ackrt ${ }^{\mathrm{tmlCcbl1}}$ mice and Dr. R. Gentek (Edinburgh University, UK) for advice regarding embryonic lineage tracing. The SNP\&SEQ Platform is part of the National Genomics Infrastructure (NGI) Sweden and Science for Life Laboratory. The SNP\&SEQ Platform is also supported by the Swedish Research Council and the Knut and Alice Wallenberg Foundation. This work was supported by grants awarded to W.W.A. from the Lundbeck foundation (R155-2014-4184), Denmark, and the Gut Cell Atlas, an initiative funded by the Leona M. and Harry B. Helmsley Charitable Trust, US.

\section{Author contributions}

The study was designed by S.I.P, S.S., and W.W.A. Experiments in Denmark were performed by S.I.P., S.S. U.M and J.J., the grafting experiments were performed by K.W. and S.I.P, experiments in Glasgow were performed by S.I.P. and A.T.A. with support from 
1034 written by S.I.P. and W.W.A after input from all authors.

1035

1036 Declaration of Interests

1037 The authors declare no competing interests.

1038

1039

1040

1041 Table 1: Primary and secondary antibodies

\begin{tabular}{|l|l|l|}
\hline Antibodies & & \\
\hline BV711 anti-mouse CD9 clone KMC8 & BD Biosciences & Cat\#740696, RRID: AB_2740380 \\
\hline AF700 anti-mouse CD11b clone M1/70 & BioLegend & Cat\#101222, RRID: AB_493705 \\
\hline AF700 anti-mouse CD11c clone N418 & BioLegend & Cat\#117320, RRID: AB_528736 \\
\hline PE anti-mouse CD26 clone H194-112 & BioLegend & Cat\#137804, RRID: AB_2293047 \\
\hline AF594 anti-mouse CD31 clone MEC13.3 & BioLegend & Cat\#102520, RRID: AB_2563319 \\
\hline AF647 anti-mouse CD31 clone MEC13.3 & BioLegend & Cat\#102515, RRID: AB_2161030 \\
\hline BV605 anti-mouse CD31 clone 390 & BioLegend & Cat\#102427, RRID: AB_2563982 \\
\hline BV650 anti-mouse CD31 clone 390 & BD Biosciences & Cat\#740483, RRID: AB_2740207 \\
\hline PerCP/Cy5.5 anti-mouse CD31 clone 390 & BioLegend & $\begin{array}{l}\text { Cat\#102420, RRID: } \\
\text { AB_10613644 }\end{array}$ \\
\hline BV421 anti-mouse CD34 clone RAM34 & BD Biosciences & $\begin{array}{l}\text { Cat\#562608, RRID: } \\
\text { AB_11154576 }\end{array}$ \\
\hline FITC anti-mouse CD34 clone RAM34 & Thermo Fischer Scientific & $\begin{array}{l}\text { Cat\#11-0341-85, RRID: } \\
\text { AB_465022 }\end{array}$ \\
\hline Rat anti-mouse CD34 clone RAM34 & Thermo Fischer Scientific & $\begin{array}{l}\text { Cat\#14-0341-82, RRID: } \\
\text { AB_467210 }\end{array}$ \\
\hline AF700 anti-mouse CD45 clone 30-F11 & Thermo Fischer Scientific & $\begin{array}{l}\text { Cat\#56-0451-82, RRID: } \\
\text { AB_891454 }\end{array}$ \\
\hline AF700 anti-mouse CD45.2 clone 104 & Thermo Fischer Scientific & $\begin{array}{l}\text { Cat\#56-0454-82, RRID: } \\
\text { AB_657752 }\end{array}$ \\
\hline APCCy7 anti-mouse CD45.2 clone 104 & BioLegend & Cat\#109824, RRID: AB_830789 \\
\hline Biotin anti-mouse CD81 clone Eat-2 & BioLegend & Cat\#104903, RRID: AB_313138 \\
\hline APCCy7 anti-mouse CD90.2 clone 53-2.1 & BD Biosciences & $\begin{array}{l}\text { Cat\#561641, RRID: } \\
\text { AB_10898013 }\end{array}$ \\
\hline FITC anti-mouse CD90.2 clone 53-2.1 & Thermo Fischer Scientific & $\begin{array}{l}\text { Cat\#11-0902-82, RRID: } \\
\text { AB_465154 }\end{array}$ \\
\hline PE anti-mouse CD141 clone REA964 & Miltenyi & $\begin{array}{l}\text { Cat\#130-116-017, RRID: } \\
\text { AB_2727308 }\end{array}$ \\
\hline BUV395 anti-mouse CD146 clone ME-9F1 & BD Biosciences & Cat\#740330, RRID: AB_2740063 \\
\hline AF488 anti-mouse $\alpha$ SMA clone 1A4 & Abcam & Cat\#ab184675, RRID: \\
AB_2832195 \\
\hline AF700 anti-mouse B220 clone RA3-6B2 & BioLegend & Cat\#103232, RRID: AB_493717 \\
\hline
\end{tabular}




\begin{tabular}{|c|c|c|}
\hline APC anti-mouse BP3 clone BP3 & BioLegend & $\begin{array}{l}\text { Cat\#140208, RRID: } \\
\text { AB_10901172 }\end{array}$ \\
\hline BV650 anti-mouse BP3 clone BP-3 & BD Biosciences & Cat\#740611, RRID: AB_2740311 \\
\hline BV786 anti-mouse BP3 clone BP-3 & BD Biosciences & Cat\#741012, RRID: AB_2740634 \\
\hline AF555 anti-mouse CXCL14 rabbit polyclonal & BIOSS & Cat\#bs-1503R-A555 \\
\hline AF647 anti-mouse EpCAM clone G8.8 & BioLegend & Cat\#118211, RRID: AB_1134104 \\
\hline APCCy7 anti-mouse EpCAM clone G8.8 & BioLegend & Cat\#118218, RRID: AB_2098648 \\
\hline BV510 anti-mouse EpCAM clone G8.8 & BD Biosciences & Cat\#563216, RRID: AB_2738075 \\
\hline PerCP-eF710 anti-mouse EpCAM clone G8.8 & Thermo Fischer Scientific & $\begin{array}{l}\text { Cat\#46-5791-82, RRID: } \\
\text { AB_10598205 }\end{array}$ \\
\hline PE anti-mouse ESAM clone 1G8 & BioLegend & Cat\#136204, RRID: AB_1953301 \\
\hline Rabbit anti-mouse FGFR2 polyclonal & Proteintech & $\begin{array}{l}\text { Cat\#13042-1-AP, RRID: } \\
\text { AB_10642943 }\end{array}$ \\
\hline AF700 anti-mouse Gr-1 clone RB6-8C5 & BioLegend & Cat\#108422, RRID: AB_2137487 \\
\hline AF488 donkey anti-rat IgG & Jackson IR & $\begin{array}{l}\text { Cat\#712-545-153, RRID: } \\
\text { AB_2340684 }\end{array}$ \\
\hline Cy3 donkey anti-rat IgG & Jackson IR & $\begin{array}{l}\text { Cat\#712-166-150, RRID: } \\
\text { AB_2340668 }\end{array}$ \\
\hline AF647 donkey anti-goat IgG & Jackson IR & $\begin{array}{l}\text { Cat\#705-605-147, RRID: } \\
\text { AB_2340437 }\end{array}$ \\
\hline AF647 donkey anti-rabbit IgG & Jackson IR & $\begin{array}{l}\text { Cat\#711-605-152, RRID: } \\
\text { AB_2492288 }\end{array}$ \\
\hline BV605 anti-mouse Itgb1 clone HM $\beta 1-1$ & BD Biosciences & Cat\#740365, RRID: AB_2740097 \\
\hline APC anti-mouse L1CAM clone 555 & Miltenyi & $\begin{array}{l}\text { Cat\#130-102-221, RRID: } \\
\text { AB_2655594 }\end{array}$ \\
\hline APC anti-mouse NCAM clone 809220 & $R \& D$ & Cat\#FAB7820A \\
\hline AF700 anti-mouse NK1.1 clone PK136 & BioLegend & Cat\#108730, RRID: AB_2291262 \\
\hline BV421 anti-mouse PDGFR $\alpha$ clone APA5 & BD Biosciences & Cat\#566293, RRID: AB_2739666 \\
\hline Goat anti-mouse PDGFR $\alpha$ polyclonal & $\mathrm{R} \& \mathrm{D}$ & $\begin{array}{l}\text { Cat\#AF1062, RRID: } \\
\text { AB_2236897 }\end{array}$ \\
\hline PE/CF594 anti-mouse PDGFR $\alpha$ clone APA5 & BD Biosciences & Cat\#562775, RRID: AB_2737786 \\
\hline PECy7 anti-mouse PDPN clone 8.1.1 & Thermo Fischer Scientific & $\begin{array}{l}\text { Cat\#25-5381-82, RRID: } \\
\text { AB_2573460 }\end{array}$ \\
\hline Rabbit anti-mouse PPAR $\gamma$ polyclonal & Invitrogen & $\begin{array}{l}\text { Cat\#PA5-25757, RRID: } \\
\text { AB_2543257 }\end{array}$ \\
\hline AF700 anti-mouse Ter119 clone Ter119 & BioLegend & Cat\#116220, RRID: AB_528963 \\
\hline APC-eF780 anti-mouse Ter119 clone Ter119 & Thermo Fischer Scientific & $\begin{array}{l}\text { Cat\#47-5921-82, RRID: } \\
\text { AB_1548786 }\end{array}$ \\
\hline BV510 streptavidin & BD Biosciences & Cat\#563261, RRID: AB_2869477 \\
\hline
\end{tabular}




\section{Supplementary Files}

This is a list of supplementary files associated with this preprint. Click to download.

- Supplementallnformation.pdf

- SupplementaryTable1.xlsx

- SupplementaryTable2.xlsx

- SupplementaryTable3.xlsx 